\title{
Associated Production of CP-odd and Charged Higgs Bosons at Hadron Colliders
}

\author{
QIng-Hong CaO ${ }^{1} *$ Shinya Kanemura ${ }^{2}+$ and and C.-P. Yuan \\ ${ }^{1}$ Department of Physics \& Astronomy, \\ Michigan State University, East Lansing, \\ Michigan 48824-1116, USA \\ ${ }^{2}$ Department of Physics, \\ Osaka University, Toyonaka, \\ Osaka 560-0043, Japan
}

\begin{abstract}
In the Minimal Supersymmetric Standard Model, the masses of the charged Higgs boson $\left(H^{ \pm}\right)$ and the CP-odd scalar $(A)$ are related by $M_{H^{+}}^{2}=M_{A}^{2}+m_{W}^{2}$ at the Born level. Because the coupling of $W^{-}-A-H^{+}$is fixed by gauge interaction, the Born level production rate of $q \bar{q}^{\prime} \rightarrow W^{ \pm *} \rightarrow A H^{ \pm}$ depends only on one supersymmetry parameter - the mass $\left(M_{A}\right)$ of $A$. We examine the sensitivity of the LHC to this signal event in the $A(\rightarrow b \bar{b}) H^{+}\left(\rightarrow \tau^{+} \nu_{\tau}\right)$ and $A(\rightarrow b \bar{b}) H^{+}(\rightarrow t \bar{b})$ decay channels. We illustrate how to test the mass relation between $A$ and $H^{+}$in case that the signal is found. If the signal is not found, the product of the decay branching ratios of $A$ and $H^{ \pm}$predicted by the MSSM is bounded from above as a function of $M_{A}$.
\end{abstract}

PACS numbers: 12.60.-i;12.15.-y;11.15.Ex

*Electronic address: cao@pa.msu.edu

$\dagger$ Electronic address: kanemu@het.phys.sci.osaka-u.ac.jp

‡Electronic address: yuan@pa.msu.edu 


\section{INTRODUCTION}

One of the top priorities of current and future high-energy colliders, such as the Fermilab Tevatron and CERN Large Hadron Collider (LHC), is to probe the mechanism of the electroweak symmetry breaking. In the Standard Model (SM) of particle physics, this amounts to searching for the yet-to-be-found Higgs boson. It is also possible that the mechanism of electroweak symmetry breaking originates from new physics beyond the SM. Very often, the low energy effective theory of new physics model predicts the existence of extended Higgs sector that contains more than one Higgs boson. Hence, in order to discriminate each new physics model from its alternatives, it is important to detect these additional Higgs bosons and to measure their properties.

Supersymmetry (SUSY) is one of the most commonly studied new physics models. The Higgs sector of the Minimal Supersymmetric Standard Model (MSSM) is known as a special

case of the Two Higgs Doublet Model (THDM) with the type-II Yukawa interaction [1]. The THDM yields five physical scalar states, i.e., two CP-even $(h$ nd $H$ ), a CP-odd $(A)$ and a pair of charged Higgs bosons $\left(H^{ \pm}\right)$.

In contrast to the free parameters in the Higgs sector of the THDM, the masses and mixing angles among the Higgs bosons in the MSSM are determined by the gauge couplings due to the requirement of supersymmetry. One of the striking features of the MSSM Higgs sector is that the mass $\left(M_{h}\right)$ of the lightest Higgs boson $h$ is predicted to be smaller than the mass of the $Z$ boson at the tree level and less than about $130 \mathrm{GeV}$ after including contribution from radiative corrections 2]. The discovery of such a light Higgs boson could be a strong hint of the MSSM, as the mass of the SM Higgs boson has to be approximately between $130 \mathrm{GeV}$ and $180 \mathrm{GeV}$ for the SM to be a valid theory all the way up to the Planck scale $\left(10^{19} \mathrm{GeV}\right)[3$, , 4]. However, the discovery of a light Higgs boson does not exclude new physics models in which the light Higgs boson naturally has a mass less than $130 \mathrm{GeV}$, even when the Planck scale is taken as the cut-off scale of the models. Two examples are the non-SUSY THDM and Zee model [5]. Therefore, a detailed study of the Higgs sector is necessary to discriminate the MSSM from other new physics models. Needless to say that a general test of the MSSM should also include the experimental identification of super-partner particles, such as sfermions, charginos and neutralinos. 
Many studies have been done in the literature to show how to detect heavier MSSM Higgs bosons produced at the LHC [6, 7]. A light charged Higgs boson with mass $M_{H^{ \pm}}<m_{t}-m_{b}$ may be produced via the top quark decay [8] or via the pair production $q q \rightarrow H^{+} H^{-}$[9], $g g \rightarrow H^{+} H^{-}$[10] and $q q \rightarrow H^{+} H^{-} q q$ [11]. The main production channels for the heavier $H^{ \pm}$may be those associated with heavy quarks such as $g b \rightarrow H^{-} t[12]$ and $q b \rightarrow q^{\prime} b H^{ \pm}\lfloor 13]$. The associated production of the $W$ boson and charged Higgs boson $b \bar{b}, g g \rightarrow H^{ \pm} W^{\mp}$ [14] may also be substantial, especially for small and large $\tan \beta$ values. The possible discovery channels from the decay of $H^{ \pm}$are $H^{ \pm} \rightarrow t b$ [15], $H^{ \pm} \rightarrow \tau \nu$ [16], and $H^{ \pm} \rightarrow W^{ \pm} h$ [17]. Furthermore, the CP-odd Higgs boson may be produced mainly in association with bottom quarks $(g g, q q \rightarrow A b \bar{b})[18]$. The possible discovery channels from the decay of $A$ are $A \rightarrow$ $t \bar{t}$ [7], $A \rightarrow \gamma \gamma$ [19] and $A \rightarrow Z h$ [20] for low $\tan \beta$ values, and $A \rightarrow \mu^{+} \mu^{-}$[21] or $\tau \bar{\tau}$ [22] for large $\tan \beta$ values. The production and decay channels discussed above can be used to probe a large part of MSSM parameter space in the $M_{A}$ versus $\tan \beta$ plane, except for intermediate values of $\tan \beta$ and large values of $M_{A}$ where only the lightest Higgs boson $h$ is likely to be found.

In a recent paper [23], two of us proposed to study the associated production of CP-odd scalar $(A)$ and charged Higgs boson $\left(H^{ \pm}\right)$via $q \bar{q}^{\prime} \rightarrow W^{ \pm *} \rightarrow A H^{ \pm}$at hadron colliders to test the MSSM. It was shown that at the Born level the production of this process depends only on one SUSY parameter - the mass $\left(M_{A}\right)$ of $A$. This is because in the MSSM the masses of $H^{ \pm}$and $A$ are related by the mass of the $W^{ \pm}$boson $\left(m_{W}\right)$ as ${ }^{1}$

$$
M_{H^{ \pm}}^{2}=M_{A}^{2}+m_{W}^{2}
$$

and the coupling of $W^{-}-A-H^{+}$is fixed by gauge interaction. In case that $M_{A}$ can be measured from data by reconstructing its decay particles (such as $b \bar{b}$ pair), the Born level production rate of this process is uniquely determined and does not depend on any other SUSY parameters. Therefore, studying this process can test the product of the Higgs boson decay branching ratios (in contrast to the product of decay branching ratios and production rate) predicted by the MSSM. The above conclusion holds for any SM decay model of $A$

\footnotetext{
1 We note that in general, a CP violating phase can enter the Higgs sector of the MSSM, so that the CP-even Higgs bosons can mix with the CP-odd Higgs scalar and the mass relation (1) does not hold any more. In this paper, we shall focus our study on the MSSM with a CP invariant Higgs sector.
} 
and $H^{ \pm}$. For example, if the decay mode of $A \rightarrow b \bar{b}$ and $H^{+} \rightarrow \tau^{+} \nu_{\tau}$ in the $A H^{+}$event is studied and no excess is found in experimental data for a given mass bin of $M_{A}$ (hence, $\left.M_{H^{ \pm}}\right)$, one can constrain the MSSM by demanding the product of the decay branching ratios, $\operatorname{Br}(A \rightarrow b \bar{b}) \times \operatorname{Br}\left(H^{+} \rightarrow \tau^{+} \nu_{\tau}\right)$, to be bounded from above as a function of $M_{A}$. Similarly, applying the same strategy, one can constrain the product $\operatorname{Br}(A \rightarrow X) \times \operatorname{Br}\left(H^{+} \rightarrow Y\right)$ for any decay modes $X$ and $Y$ predicted by the MSSM, as a function of only one SUSY parameter $-M_{A}$.

Another interesting feature of this process is that the kinematic acceptance (therefore, the detection efficiency) of the signal events does not depend on the choice of the other SUSY parameters because both $A$ and $H^{ \pm}$are spin-0 (pseudo-)scalar particles so that the kinematic distributions of their decay particles can be accurately modelled. (It is an isotropic distribution in the rest frame of $A$ or $H^{ \pm}$.) Moreover, the one-loop electroweak corrections to the mass relation (II) and the $W^{-} A H^{+}$coupling are generally smaller than the other theoretical errors (such as the parton distribution function uncertainties) and the expected experimental errors (such as the mass resolution of Higgs boson decaying into jets). Despite that for some extreme choice of the MSSM parameters, the mass relation (II) can receive a relatively large correction [24], its effect to the $A H^{+}$production rate is found to be small. Therefore, in this work we shall only perform a Born level Monte Carlo analysis to study the sensitivity of the LHC to this production channel.

To detect the signal event at hadron colliders, it is necessary to suppress the potentially large background rates predicted by the SM. In this paper, we show how to detect such a signal at the LHC for the specific decay mode of $A H^{ \pm} \rightarrow b \bar{b} \tau^{+} \nu_{\tau}$ with $\tau^{+} \rightarrow \pi^{+} \bar{\nu}_{\tau}$. While it is possible to include other decay channels of $A H^{ \pm}$, we shall restrict ourselves to this simple decay mode and use it as an example to illustrate the idea of Ref. [23]. Namely, studying the associated production of $A$ and $H^{ \pm}$at hadron colliders can discriminate the MSSM from its alternatives, e.g., a general two-Higgs-doublet model (THDM), and test the prediction of the MSSM on the product of the decay branching ratios of $A$ and $H^{ \pm}$.

The rest of the paper is organized as follows. In Sec. II, we briefly review the result of Ref. 23] for the production rate of the signal event at the Fermilab Tevatron and CERN LHC up to the one-loop order QCD and electroweak corrections. In Sec. III, we present a 


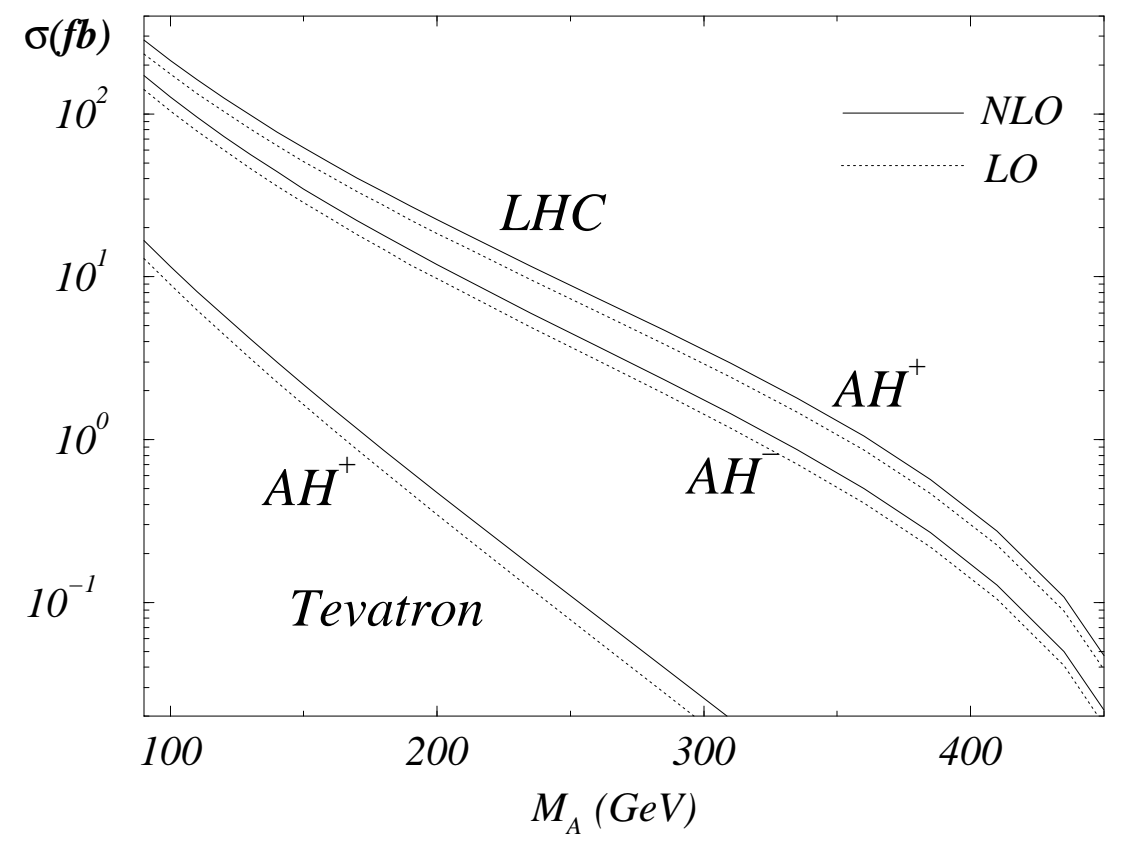

FIG. 1: The LO (dotted lines) and NLO QCD (solid lines) cross sections of the $A H^{+}$and $A H^{-}$ pairs as a function of $M_{A}$ at the Tevatron (a $1.96 \mathrm{TeV} p \bar{p}$ collider), and the LHC (a $14 \mathrm{TeV} p p$ collider). The cross sections for $A H^{+}$and $A H^{-}$pair productions coincide at the Tevatron for being a $p \bar{p}$ collider.

Monte Carlo analysis for studying the sensitivity of the LHC to the detection of the signal event. We show that all the dominant SM backgrounds can be effectively suppressed by making use of the polarization of $\tau^{ \pm}$. Sec. IV contains our conclusions.

\section{PRODUCTION CROSS SECTION OF SIGNAL}

At hadron colliders, the Born level process for the production of a $A H^{ \pm}$pair is $q \bar{q}^{\prime} \rightarrow$ $W^{ \pm *} \rightarrow A H^{ \pm}$. The coupling of $W^{\mp}-A-H^{ \pm}$is induced from the gauge invariant kinetic term of the Higgs sector [1]:

$$
\mathcal{L}_{\text {int }}=\frac{g}{2} W_{\mu}^{+}\left(A \partial^{\mu} H^{-}-H^{-} \partial^{\mu} A\right)+\text { h.c. },
$$

and its strength is determined by the weak gauge coupling $g$. Hence, the Born level cross section $\sigma\left(p \bar{p}, p p \rightarrow A H^{ \pm}\right)$depends only on the masses of $A$ and $H^{ \pm}$. Its squared amplitude, 
after averaging over the spins and colors is

$$
\overline{|\mathcal{M}|^{2}}=\frac{4}{3} m_{W}^{4} G_{F}^{2} \frac{s}{\left(s^{2}-m_{W}^{2}\right)^{2}+m_{W}^{2} \Gamma_{W}^{2}} P^{2} \sin ^{2} \theta
$$

where $P=\sqrt{E_{A}^{2}-M_{A}^{2}}$ with $E_{A}=\left(s+M_{A}^{2}-M_{H^{+}}^{2}\right) /(2 \sqrt{s})$ and $\theta$ is the polar angle of $A$ in the center-of-mass (c.m.) frame of $A$ and $H^{ \pm}$. In the MSSM, the masses of the charged Higgs boson $H^{ \pm}$and the CP-odd scalar $A$ are strongly correlated. At the Born level, $M_{H^{ \pm}}=\sqrt{M_{A}^{2}+m_{W}^{2}}$. In Fig. 1, we show the inclusive production rate of $A H^{ \pm}$as a function of $M_{A}$ for the Tevatron (a $1.96 \mathrm{TeV}$ proton-antiproton collider) and the LHC (a $14 \mathrm{TeV}$ proton-proton collider). Here, the CTEQ6M parton distribution functions (PDF) 25] are used and both the renormalization and the factorization scales are chosen to be the invariant mass $(\sqrt{s})$ of the $A H^{ \pm}$pair. We note that for the $c \bar{b} \rightarrow A H^{+}$subprocess, in addition to the Cabbibo-Kobayashi-Maskawa suppressed $s$-channel $W$-boson diagram, there is a $t$-channel diagram whose contribution becomes large for a large value of $\tan \beta$. Nevertheless, the $c \bar{b} \rightarrow A H^{+}$contribution to the inclusive $A H^{+}$rate is small. For example, its contribution to the total rate is less than $0.01 \%$ and $0.1 \%$ at the Tevatron and the LHC, respectively, for $\tan \beta=40$ and $M_{A}=90 \mathrm{GeV}$. For a smaller value of $\tan \beta$, its contribution becomes negligible. Hence, we shall ignore its contribution in the following discussion.

It is easy to include the next-to-leading order (NLO) QCD correction to calculate the production rate of the signal event because at the one-loop order only the initial state receives radiative contribution which is similar to the NLO QCD correction to the $W$-boson production, but with a different invariant mass, at hadron colliders. The result is also shown in Fig. 1. We find that typically the NLO QCD production rate is about $20 \%$ higher than the leading-order (LO) rate when the same PDF is used. The higher order ( $\alpha_{s}^{2}$ or above) QCD correction is estimated to be about $10 \%$ at the Tevatron and less than a percent at the LHC, for $M_{A}=120 \mathrm{GeV}$, when the factorization scale is varied around the $A H^{ \pm}$invariant mass $\sqrt{s}$ by a factor of 2 .

The dominant one-loop electroweak corrections to the $q \bar{q}^{\prime} \rightarrow A H^{ \pm}$process come from the loops of top $(t)$ and bottom $(b)$ quarks as well as their supersymmetric partners, i.e. stops $\left(\tilde{t}_{1,2}\right)$ and sbottoms $\left(\tilde{b}_{1,2}\right)$, in the MSSM, due to their potentially large couplings to Higgs bosons.

In the following, we discuss the quark- and squark-loop radiative corrections to the ef- 
fective coupling of $W^{ \pm} A H^{\mp}$ and to the mass relation (11). In our calculation, we adopt the on-shell renormalization scheme developed by Dabelstein in Ref. [26]. (For completeness, we outline the renormalization scheme in Appendix A).

The part of the one-loop effective coupling of $W^{ \pm} A H^{\mp}$, that is relevant to the production process $q \bar{q}^{\prime} \rightarrow H^{+} A$, can be written as $^{2}$

$$
M_{W H A}^{\mu}\left(q^{2}\right)=-\frac{\bar{g}}{2}\left(p_{A}-p_{H}\right)^{\mu}\left[1+F^{(1)}\left(q^{2}\right)\right]
$$

where $q^{\mu}, p_{A}^{\mu}$ and $p_{H}^{\mu}$ are the incoming momenta of $W^{+}, A$ and $H^{-}$, respectively, and $\bar{g}$ is the effective weak gauge coupling evaluated at $q^{2}$. Hence, the radiative correction to the cross section of the sub-process $q \bar{q}^{\prime} \rightarrow \mathrm{AH}^{+}$at the one-loop order is

$$
K^{(1)}\left(q^{2}\right) \equiv 2 \operatorname{Re} F^{(1)}\left(q^{2}\right)
$$

where the detailed calculation for $F^{(1)}\left(q^{2}\right)$ is summarized in Appendix B. As shown in Eqs. (28), (56) and (157), the quark-loop contribution is proportional to the squared Yukawa coupling constants $y_{t}^{2}\left(=2 m_{t}^{2} \cot ^{2} \beta / v^{2}\right)$ and $y_{b}^{2}\left(=2 m_{b}^{2} \tan ^{2} \beta / v^{2}\right)$. In the large $m_{t}$ or large $m_{b} \tan \beta$ limit, it can be written as

$$
F_{\text {quark }}^{(1)} \sim \frac{N_{c}}{16 \pi^{2}}\left[-\frac{1}{4} y_{t}^{2}+\frac{1}{2}\left(\frac{3}{2}-\ln \frac{m_{t}^{2}}{m_{b}^{2}}\right) y_{b}^{2}\right],
$$

where $N_{c}(=3)$ is the number of colors. Since $y_{t}^{2}$ and $y_{b}^{2}$ are at most $\mathcal{O}(1)$ for $\tan \beta \simeq 1$ and $m_{t} / m_{b}$, respectively, $F_{\text {quark }}^{(1)}$ is at most a few percent for $1 \lesssim \tan \beta \lesssim m_{t} / m_{b}$.

We also calculate the squark-loop contribution. As compared to the quark-loop effects, the squark-loop effects are rather complex because of the additional SUSY parameter dependence. The mass eigenstates $\tilde{f}_{1,2}(\tilde{f}=\tilde{t}$ or $\tilde{b})$ of the squarks are obtained from the weak eigenstates $\tilde{f}_{L, R}$ by diagonalizing the mass matrices defined through [27]

$$
\mathcal{L}_{\text {mass }}=-\left(\tilde{f}_{L}^{*}, \tilde{f}_{R}^{*}\right)\left(\begin{array}{cc}
M_{L}^{2} & m_{f} X_{f} \\
m_{f} X_{f} & M_{R}^{2}
\end{array}\right)\left(\begin{array}{c}
\tilde{f}_{L} \\
\tilde{f}_{R}
\end{array}\right),
$$

where, $M_{L}^{2}=M_{\tilde{Q}}^{2}+m_{f}^{2}+\left(m_{Z}^{2} \cos 2 \beta\right)\left(T_{f_{L}}-Q_{f} s_{W}^{2}\right)$ and $M_{R}^{2}=M_{\tilde{U}, \tilde{D}}^{2}+m_{f}^{2}+\left(m_{Z}^{2} \cos 2 \beta\right) Q_{f} s_{W}^{2}$. In this expression, $M_{\tilde{Q}}^{2}$, $M_{\tilde{U}}^{2}($ for $\tilde{f}=\tilde{t})$ and $M_{\tilde{D}}^{2}$, (for $\left.\tilde{f}=\tilde{b}\right)$ are the soft-breaking masses for

\footnotetext{
${ }^{2}$ The other form factor, $\left(p_{A}+p_{H}\right)^{\mu}$, does not contribute to this process for massless quarks.
} 
$\tilde{f}_{L}, \tilde{t}_{R}$ and $\tilde{b}_{R}$, respectively; $s_{W}=\sin \theta_{W}$ with $\theta_{W}$ being the weak mixing angle; $T_{f_{L}}$ is the isospin of the left-handed quark $f_{L}$; and $Q_{f}$ is the electric charge of the quark $f$. Moreover, $X_{t}=A_{t}-\mu \cot \beta$ and $X_{b}=A_{b}-\mu \tan \beta$, where $A_{t}\left(A_{b}\right)$ is the trilinear $A$-term for $\tilde{t}(\tilde{b})$, and $\mu$ is the SUSY invariant higgsino mass parameter [27]. For completeness, we have listed all the relevant squark and Higgs boson couplings in Appendix C, so that the squark-loop contributions to $F^{(1)}(s)$, cf. Eq. (26), can be directly read out from the results in Eqs. (30), (58) and (59).

To examine the effect of one-loop electroweak corrections, we shall discuss two limiting cases below. Firstly, we consider the cases with $\mu=A_{t}=A_{b}=0$, i.e., the cases without stop mixing $\left(\left|X_{t}\right|=0\right)$ and sbottom mixing $\left(\left|X_{b}\right|=0\right)$. Under this scenario, the masses of squarks are proportional to the typical scale of the soft-breaking mass, denoted as $M$. Since all the relevant couplings between squarks and Higgs bosons are independent of the soft-breaking masses $M_{\tilde{Q}}, M_{\tilde{U}}$ and $M_{\tilde{D}}$ (see Appendix $\mathbf{C}$ ), the squark-loop effect is decoupled and its contribution is very small for a large value of $M$, where $M \equiv M_{\tilde{Q}} \simeq M_{\tilde{U}} \simeq M_{\tilde{D}}$. For a smaller $M, F_{\text {squark }}^{(1)}$ becomes larger. However, $M$ cannot be too small because a small $M$ implies light squarks whose masses are already bounded from below by the direct search results [28]. Furthermore, as to be shown later, the case with a small $M$ is also strongly constrained by the $\rho$ parameter measurement. Secondly, we examine the case with a large stop mixing, assuming $m_{t}\left|X_{t}\right| \sim M^{2} \gg m_{Z}^{2}$. Such a large stop mixing leads to a large mass splitting between $\tilde{t}_{1}$ and $\tilde{t}_{2}$ so that $m_{\tilde{t}_{1}} \simeq \mathcal{O}\left(m_{Z}\right)$ and $m_{\tilde{t}_{2}} \simeq \sqrt{2} M$, while $m_{\tilde{b}_{1,2}} \simeq M$. The leading squark contribution to $F^{(1)}\left(q^{2}\right)$ can be expressed as

$$
F_{\text {squark }}^{(1)} \sim \frac{-N_{c}}{16 \pi^{2}}\left[\left(\frac{3}{4}-\ln 2\right)\left(\frac{Y_{\tilde{t}}}{M}\right)^{2}+\left(\frac{13}{6}-3 \ln 2\right)\left(\frac{Y_{\tilde{b}}}{M}\right)^{2}\right],
$$

with $Y_{\tilde{t}}=\frac{m_{t}}{v}\left(A_{t} \cot \beta+\mu\right)$ and $Y_{\tilde{b}}=\frac{m_{b}}{v}\left(A_{b} \tan \beta+\mu\right)$. Since in this case $\left|A_{t}\right| \simeq \mid M^{2} / m_{t} \pm$ $|\mu| \cot \beta \mid$, we have $\left|Y_{\hat{t}}\right| \lesssim \mathcal{O}\left(M^{2} / v\right)$ for $|\mu| \lesssim M$ and $\tan \beta \gtrsim 1$. When $\left|A_{b}\right| \simeq\left|A_{t}\right|>|\mu|$ and $\tan \beta \lesssim m_{t} / m_{b}$, we find $\left|Y_{\tilde{b}}\right| \lesssim \mathcal{O}\left(M^{2} / v\right)$. Thus, with a large stop mixing $\left(m_{t}\left|X_{t}\right| \simeq M^{2}\right)$, $F_{\text {squark }}^{(1)}$ is proportional to the soft-breaking mass scale $M$, and does not decouple in the large $M$ limit. However, such kind of model is strongly constrained by the $\rho$-parameter (or the $T$-parameter) data. With a large stop mixing $\left(M^{2} \simeq m_{t}\left|X_{t}\right|\right)$, the squark contribution to 
the $\rho$-parameter (cf. Appendix D) is

$$
\Delta \rho_{\text {squark }} \simeq\left(2.2 \times 10^{-3}\right) \frac{M^{2}}{v^{2}} .
$$

Since any new physics contribution to the $\rho$-parameter has to be bounded by data as 29]

$$
-1.7<\Delta \rho_{\text {new }} \times 10^{3}<2.7, \quad \text { at } 2 \sigma \text { level }
$$

the scale $M$ cannot be too large in this case. Consequently, the above $F_{\text {squark }}^{(1)}$ is constrained to be smaller than a few percent as long as $\mu^{2}$ is not much larger than $M^{2}$.

To examine the effect from the stop and sbottom loops to the production rate of $A H^{ \pm}$, we consider 4 sets of SUSY parameters, as listed in Table \ which give the largest allowed deviation in the $\rho$-parameter. Set 1 and Set 2 represent the cases without either a stop mixing $\left(X_{t}=0\right)$ or a sbottom mixing $\left(X_{b}=0\right)$, and Set 3 and Set 4 are the cases with a large stop mixing $\left(m_{t}\left|X_{t}\right| \simeq M^{2}\right)$ and $m_{\tilde{t}_{1}} \simeq 100 \mathrm{GeV}$. The $K^{(1)}(s)$ factor, as defined in Eq. (5), is shown in Fig. 2 as a function of the invariant mass $(\sqrt{s})$ of the constituent process for $M_{A}=90 \mathrm{GeV}$. It is clear that the quark-loop contribution to $K^{(1)}(s)$ dominates the squarkloop contribution. For these four sets of SUSY parameters, the squark-loop contribution is smaller than the quark-loop contribution by about a factor of 100 . Generally, the squark contributions are at most a few percent, unless $|\mu|$ is taken to be very large as compared to the scale $M$. We have checked that this conclusion does not change when the assumption $M_{Q}^{2} \simeq M_{U}^{2} \simeq M_{D}^{2}$ is relaxed to some extent. Including both the quark- and squark-loop contributions to $K^{(1)}(s)$, we found that the correction to the hadronic cross section of $H^{+} A$ production in the invariant mass region just above the $H^{+} A$ threshold, where the constituent cross section is the largest, is at a percent level. In summary, to be consistent with the lowenergy data and the direct search results for stops and sbottoms, the one-loop electroweak correction to the production rate of $p p, p \bar{p} \rightarrow A H^{ \pm}$is small (at most a few percent).

Next, we discuss the one-loop corrections to the mass relation (11). Let us parameterize the deviation from the tree-level relation by $\delta$, so that at the one-loop order

$$
M_{H^{ \pm}}=\sqrt{M_{A}^{2}+m_{W}^{2}}(1+\delta) .
$$

We note that in our renormalization scheme (see Appendix $\mathbf{A}$ ), $M_{A}$ and $m_{W}$ are the input parameters, but $M_{H^{+}}$is not. The one-loop corrected mass of the charged Higgs boson $M_{H^{ \pm}}$ 
TABLE I: The SUSY (input and output) parameters used in Fig. 2

\begin{tabular}{c|cccc}
\hline \hline & Set1 & Set2 & Set 3 & Set4 \\
\hline$M_{\tilde{Q}}=M_{\tilde{U}}=M_{\tilde{D}}(\mathrm{GeV})$ & 106 & 84 & 408 & 409 \\
$\tan \beta$ & 2 & 40 & 2 & 40 \\
$A_{t}=A_{b}(\mathrm{GeV})$ & 0 & 0 & +1261 & +1119 \\
$\mu$ & 0 & 0 & +300 & +300 \\
\hline \hline$m_{\tilde{t}_{1}}(\mathrm{GeV})$ & 197 & 184 & 100 & 100 \\
$m_{\tilde{t}_{2}}(\mathrm{GeV})$ & 199 & 188 & 612 & 611 \\
$m_{\tilde{b}_{1}}(\mathrm{GeV})$ & 108 & 88 & 407 & 373 \\
$m_{\tilde{b}_{2}}(\mathrm{GeV})$ & 116 & 103 & 412 & 447 \\
\hline \hline$\rho_{\text {squarks }} \times 10^{3}$ & 2.72 & 2.70 & 2.71 & 2.70 \\
\hline \hline
\end{tabular}

can be obtained by solving

$$
0=\operatorname{Det}\left|\begin{array}{lll}
\Gamma_{G^{+} G^{-}}^{(2)}\left(p^{2}\right) & \Gamma_{G^{+} H^{-}}^{(2)}\left(p^{2}\right) \\
\Gamma_{H^{+} G^{-}}^{(2)}\left(p^{2}\right) & \Gamma_{H^{+} H^{-}}^{(2)}\left(p^{2}\right)
\end{array}\right|
$$

where $\Gamma_{i j}^{(2)}\left(p^{2}\right)$ represent the renormalized two-point functions in the basis of the renormalized Goldstone boson $\left(G^{ \pm}\right)$and charged Higgs boson $\left(H^{ \pm}\right)$fields. Here, the notation "Det" denotes taking the determinant of the $2 \times 2$ matrix. One of the solutions of the above equation is $p^{2}=0$, which corresponds to the charged Nambu-Goldstone mode, and another is $M_{H^{ \pm}}^{2}$. At the one-loop level, the pole mass of the charged-Higgs boson, in our renormalization scheme, can be calculated from

$$
\begin{aligned}
M_{H^{ \pm}}^{2}= & M_{A}^{2}+m_{W}^{2} \\
& +\Pi_{A A}\left(M_{A}^{2}\right)-\Pi_{H^{+} H^{-}}\left(M_{A}^{2}+m_{W}^{2}\right)+\Pi_{W W}\left(m_{W}^{2}\right),
\end{aligned}
$$

where $\Pi_{\phi \phi}\left(q^{2}\right)\left(\phi=A, H^{ \pm}\right.$, and $\left.W\right)$ are the self-energies. For completeness, we summarize the quark and squark contributions to the self-energies of $A$ and $H^{ \pm}$in Appendix E.

When $A_{t, b}$ and $\mu$ are zero (i.e., no-mixing case), the leading contribution to $\delta$ is found to 


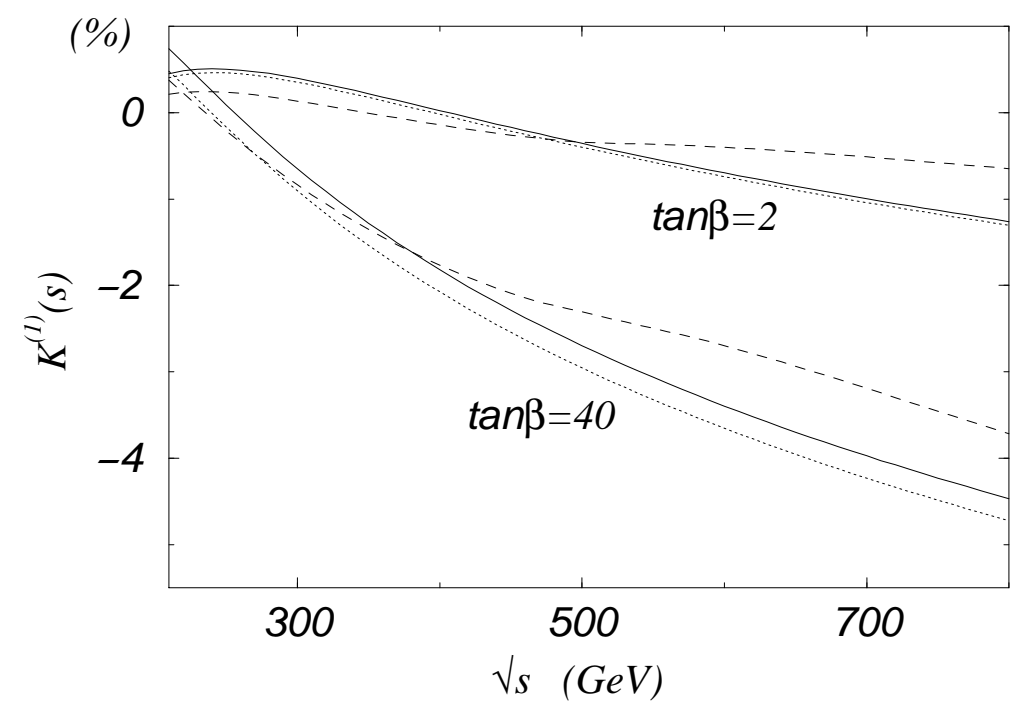

FIG. 2: The $K$-factor, $K^{(1)}(s)$, of the constituent process $q \bar{q}^{\prime} \rightarrow H^{+} A$ for $M_{A}=90 \mathrm{GeV}$, as a function of the invariant mass $\sqrt{s}$ of $q \bar{q}^{\prime}$. The solid lines correspond to the top and bottom quark contribution. The cases where the squark-loop contribution is included are described by the dotted lines for those without stop mixing (Set 1 and Set 2) and by dashed lines for those with maximal stop mixing (Set 3 and Set 4), respectively.

be

$$
\delta \sim \frac{N_{c}}{8 \pi^{2} v^{2}}\left(\frac{m_{t}^{2} m_{b}^{2}}{M_{A}^{2}+m_{W}^{2}}\right) \frac{1}{\sin ^{2} \beta \cos ^{2} \beta}\left(1+\ln \frac{M^{2}}{m_{t}^{2}}\right) .
$$

This correction is substantial for $\tan \beta \simeq m_{t} / m_{b}$ and $M^{2} \gg m_{t}^{2}$. Applying Eq. (13) with the complete expression of $\Pi_{\phi \phi}\left(q^{2}\right)$, we found $\delta$ to be less than $4.9 \%$ for $2<\tan \beta<40$, $M<2 \mathrm{TeV}$ and $M_{A}>90 \mathrm{GeV}$. Our result agrees well with Ref. [30], in which an approximate formula was presented for $M^{2} \gg m_{t}^{2}$.

For the cases with nonzero $A_{b, t}$ and $\mu$ parameters, $\delta$ receives extra contributions, which are proportional to $A_{t, b}^{4} / M^{4}, A_{t, b}^{2} \mu^{2} / M^{4}$ and $\mu^{4} / M^{4}$ [30, 31] originated from the squark couplings [cf. Eqs. (36),$(37)$, (40) and (41)] and squark masses. For the Set 3 and Set 4 parameters listed in Table प $\delta$ is found to be less than $5.3 \%$ and $3.6 \%$ for $M_{A}>90 \mathrm{GeV}$, respectively. In summary, as long as $\left|A_{t, b}\right|$ and $|\mu|$ are not too large as compared to $M$, in a wide range of the parameter space that is allowed by the available experimental and theoretical constraints, $\delta$ does not exceed 10\%. (In some corner of SUSY parameter space, $\delta$ 
can be larger than $10 \%$ 24]. Nevertheless, the $A H^{+}$production rate at the LHC will not be strongly modified as long as $M_{A}$ is not too large so that the mass threshold effect becomes important.)

Supported by the finding that the one-loop electroweak corrections to the $W^{ \pm} A H^{\mp}$ coupling and to the mass relation $M_{H^{ \pm}}^{2}=M_{A}^{2}+m_{W}^{2}$ are generally smaller than the other theoretical errors (such as the parton distribution function uncertainties) and the expected experimental errors (such as the mass resolution of Higgs boson decaying into jets, which is about $10 \mathrm{GeV}$ for a $100 \mathrm{GeV}$ Higgs boson), we conclude that studying the process $p \bar{p}, p p \rightarrow W^{ \pm *} \rightarrow A H^{ \pm}$allows us to distinguish the MSSM from its alternatives by verifying the mass relation (11), or Eq. (11), when the signal is found. If the signal is not found, studying this process can provide an upper bound on the product of the decay branching ratios of $A$ and $H^{ \pm}$as a function of only one SUSY parameter $-M_{A}$.

\section{MONTE CARLO ANALYSIS}

In this section we discuss how to detect such a signal at hadron colliders. As a concrete example, we examine the specific decay channel of $A H^{ \pm} \rightarrow b \bar{b} \tau^{+} \nu_{\tau}$ with $\tau^{+} \rightarrow \pi^{+} \bar{\nu}_{\tau}$. As shown in Fig. 1, the signal cross section at the Tevatron is much smaller than that at the LHC. Hence, we shall first discuss its phenomenology at the LHC, and then comment on the result at the Tevatron. Furthermore, since the NLO QCD and electroweak corrections to the signal cross section are small, for simplicity, we shall perform the Monte Carlo analysis at the Born level.

In the MSSM, the mass of the heavier CP-even Higgs boson $(H)$ is about the same (approximately within $10 \mathrm{GeV}$ ) as $M_{A}$ for $M_{A} \gtrsim 120 \mathrm{GeV}$ and $\tan \beta \gtrsim 10$. In this case, $q \bar{q}^{\prime} \rightarrow H H^{ \pm}$can produce similar final states as $q \bar{q}^{\prime} \rightarrow A H^{ \pm}$. In contrast to the coupling of $W^{ \pm} A H^{\mp}$ whose strength is $\frac{g}{2}$, the tree level coupling of $W^{ \pm} H H^{\mp}$ has the strength of $\frac{g}{2} \sin (\beta-\alpha)$, though with the same momentum dependence. Since $\sin ^{2}(\beta-\alpha) \simeq 1$ for $M_{A} \gtrsim 190 \mathrm{GeV}$ and $\tan \beta \gtrsim 10$, the production rate of $H H^{ \pm}$is almost the same as $A H^{ \pm}$in the decoupling limit. Thus, for a large $M_{A}$, when both of them decay into the same decay channels, it will be very difficult to separate the production of $A H^{ \pm}$from $H H^{ \pm}$unless a fine mass resolution can be achieved experimentally. Nevertheless, studying different decay 
channels can help to separate these two production modes. For example, a heavy $H$ can decay into a $Z Z$ pair at the Born level, but $A$ cannot. In the following analysis, we shall consider both the $A H^{ \pm}$and $H H^{ \pm}$production channels that contribute to the same final state.

Either in the MSSM or the Type-II THDM, for a large $\tan \beta$ value, the dominant decay mode is $h, H, A \rightarrow b \bar{b}$ for the neutral Higgs bosons $(h, H, A)$, and $H^{+} \rightarrow \tau^{+} \nu$ for a charged Higgs boson whose mass is smaller than the top quark mass. Due to the missing energy carried away by the final state neutrino, it is not possible to directly reconstruct the mass of $\mathrm{H}^{+}$in the decay mode $\mathrm{H}^{+} \rightarrow \tau^{+} \nu$. But, the transverse mass of the charged Higgs boson can be reconstructed from the $\tau$ jet momentum and missing transverse energy [16]. For $m_{H^{ \pm}} \gtrsim 200 \mathrm{GeV}$, the dominant decay mode is $H^{+} \rightarrow t \bar{b}$, in which $M_{H^{+}}$can be reconstructed after properly choosing the longitudinal momentum of the neutrino (with a two-fold solution) from $t$ decay. To cover both decay modes in our study, we consider the following three benchmark cases with $\tan \beta=40:(i) M_{A}=101 \mathrm{GeV}$ (and $M_{H^{+}}<m_{t}+m_{b}$ ), and $H^{+} \rightarrow \tau \nu$ being the dominant decay mode; $(i i) M_{A}=166 \mathrm{GeV}$ (and $M_{H^{+}} \sim m_{t}+m_{b}$ ), and $H^{+} \rightarrow \tau \nu$ being the dominant decay mode; (iii) $M_{A}=250 \mathrm{GeV}$ and $H^{+} \rightarrow t \bar{b}$ being the dominant decay mode.

\section{A. $M_{A}=101 \mathrm{GeV}$}

Taking $M_{A}=101 \mathrm{GeV}, \tan \beta=40$, and all the other SUSY parameters (soft-breaking masses and $\mu$-parameter) to be $500 \mathrm{GeV}$, one can calculate the masses, widths and decay branching ratios of the Higgs bosons using the numerical program HDECAY [32]. We find that $M_{h}=97 \mathrm{GeV}, M_{H}=113 \mathrm{GeV}$ and $M_{H^{ \pm}}=126 \mathrm{GeV}$, and the relevant decay branching ratios are $B(A \rightarrow b \bar{b})=0.91, B(H \rightarrow b \bar{b})=0.90, B\left(H^{+} \rightarrow \tau^{+} \nu\right)=0.98$, and $B\left(\tau^{+} \rightarrow \pi^{+} \bar{\nu}\right)=0.11$. The relevant total decay widths are $\Gamma_{A}=3.7 \mathrm{GeV}, \Gamma_{H}=0.38 \mathrm{GeV}$ and $\Gamma_{H^{ \pm}}=0.43 \mathrm{GeV}$. For clarity, we summarize the above results in Table II. In spite of the small $M_{h}$, this set of SUSY parameters is compatible with CERN LEP data in searching for neutral Higgs bosons of the MSSM [33], and yields $\sin (\beta-\alpha)=0.33$. 
TABLE II: The mass, width, production rate and decay branching ratio of Higgs bosons used in the Monte Carlo analysis. Here, $\tan \beta=40$, and all the other SUSY parameters (soft-breaking masses and $\mu$-parameter) are taken to be $500 \mathrm{GeV}$.

\begin{tabular}{c|c|c|c}
\hline Sets & A & B & C \\
\hline \hline$m_{A} / \Gamma_{A}(\mathrm{GeV})$ & $101 / 3.7$ & $166 / 5.6$ & $250 / 7.9$ \\
\hline$m_{h} / \Gamma_{h}(\mathrm{GeV})$ & $97 / 3.3$ & $112 / 0.04$ & $112 / 0.01$ \\
\hline$m_{H} / \Gamma_{H}(\mathrm{GeV})$ & $113 / 0.38$ & $163 / 5.5$ & $248 / 7.8$ \\
\hline$m_{H^{+}} / \Gamma_{H^{+}}(\mathrm{GeV})$ & $126 / 0.43$ & $182 / 0.68$ & $261 / 4.2$ \\
\hline$\sigma\left(A H^{+}\right) f b$ & 164 & 36.5 & 5.4 \\
\hline$\sigma\left(H H^{+}\right) f b$ & 15.0 & 37.2 & 5.4 \\
\hline$B(A \rightarrow b \bar{b})$ & 0.91 & 0.90 & 0.89 \\
\hline$B(H \rightarrow b \bar{b})$ & 0.90 & 0.90 & 0.89 \\
\hline$B\left(H^{+} \rightarrow \tau^{+} \nu\right)$ & 0.98 & 0.90 & 0.21 \\
\hline$B\left(\tau^{+} \rightarrow \pi^{+} \nu\right)$ & 0.11 & 0.11 & 0.11 \\
\hline$B\left(H^{+} \rightarrow t \bar{b}\right)$ & 0.00 & 0.09 & 0.79 \\
\hline$B\left(t \rightarrow b e^{+} \nu\right)$ & 0.11 & 0.11 & 0.11 \\
\hline $\sin (\beta-\alpha)$ & 0.33 & 0.997 & 0.999 \\
\hline \hline
\end{tabular}

\section{Signal and background Processes}

In this case, the signal event is produced via

$$
\begin{aligned}
& q+q^{\prime} \rightarrow A(\rightarrow b \bar{b}) H^{+}\left(\rightarrow \nu \tau^{+}\left(\rightarrow \pi^{+} \bar{\nu}\right)\right) \\
& q+q^{\prime} \rightarrow H(\rightarrow b \bar{b}) H^{+}\left(\rightarrow \nu \tau^{+}\left(\rightarrow \pi^{+} \bar{\nu}\right)\right) .
\end{aligned}
$$

The experimental signature of the signal events is the production of $\pi^{+} b \bar{b}$ associated with a large missing transverse momentum carried away by neutrino and anti-neutrino. In this study we shall assume that both $b$ and $\bar{b}$ jets in the signal event can be tagged with a total efficiency of $50 \%$, so that the four dominant SM background processes are:

$$
W b \bar{b}: \quad q q^{\prime} \rightarrow b \bar{b} W^{+}, W^{+} \rightarrow \tau^{+} \nu, \tau^{+} \rightarrow \pi^{+} \bar{\nu}
$$




$$
\begin{aligned}
t \bar{b}: & q q^{\prime} \rightarrow W^{*} \rightarrow t \bar{b}, t \rightarrow b W^{+}, W^{+} \rightarrow \tau^{+} \nu, \tau^{+} \rightarrow \pi^{+} \bar{\nu}, \\
W g: & q g \rightarrow q^{\prime} t \bar{b}, t \rightarrow W^{+} b, W^{+} \rightarrow \tau^{+} \nu, \tau^{+} \rightarrow \pi^{+} \bar{\nu} \\
t \bar{t}: & q q, \quad g g \rightarrow t \bar{t}, t \rightarrow W^{+} b, W^{+} \rightarrow \tau^{+} \nu, \tau^{+} \rightarrow \pi^{+} \bar{\nu}, \bar{t} \rightarrow \bar{b} W^{-} .
\end{aligned}
$$

The first two processes are the intrinsic background processes generated by the SM, and yield the same experimental signature as the signal process at the parton level. In addition to the $W b \bar{b}$ and $t \bar{b}$ processes, the other two important SM background processes are $W g$ and $t \bar{t}$. When the forward jet $\left(q^{\prime}\right)$ in the $W g$ fusion process is not detected (either falling into a large rapidity region or carrying a too small transverse momentum to be detected), it will mimic the experimental signature of the signal event. Similarly, when the decay products of $W^{-}$in $\bar{t} \rightarrow \bar{b} W^{-}$escape detection, the $t \bar{t}$ event yields the same event signature as the signal. To study how often this situation happens, we separately consider the leptonic and hadronic decay modes of $W^{-}$at the parton level. It is straightforward to study the decay modes $W^{-} \rightarrow \ell^{-} \bar{\nu}_{\ell}$ with $\ell^{-}=e^{-}$and $\mu^{-}$. For $W^{-} \rightarrow \tau^{-} \bar{\nu}_{\tau}$, we consider the decay of $\tau^{-}$into the $\ell^{-} \bar{\nu}_{\ell} \bar{\nu}_{\tau}, \pi^{-} \bar{\nu}_{\tau}$ or $\rho^{-} \bar{\nu}_{\tau}$ mode. We find that the dominant contribution after imposing the veto cuts (to veto events with extra charged lepton with large transverse momentum or hadronic activities in the central rapidity region) comes from $W^{-} \rightarrow \ell^{-} \bar{\nu}_{\ell}$ and $W^{-} \rightarrow \bar{\nu}_{\tau} \tau^{-}\left(\rightarrow \ell^{-} \bar{\nu}_{\ell} \bar{\nu}_{\tau}\right)$, with $\ell^{-}=e^{-}$and $\mu^{-}$. Since in the end of analysis, the $t \bar{t}$ rate is smaller than the $W b \bar{b}$ rate by more than one order of magnitude, we shall defer the detailed discussion on this part of the analysis to Sec. III.C, where Case C is considered. As to be shown later, for Case C, the dominant background comes from the $t \bar{t} b \bar{b}$ events which mimic the signal event signature when the decay products of the extra $W^{-}$, from $\bar{t}$ decay, escape detection.

\section{Analysis}

a. Basic cuts: To compare the relevant background event rates to the signal event rate, we shall assume the integrated luminosity of the LHC to be $100 \mathrm{fb}^{-1}$, and require the transverse momentum $\left(p_{T}^{q}\right)$ and the rapidity $\left(\eta^{q}\right)$ of $b, \bar{b}$ and $\pi^{+}$to satisfy the following basic cuts:

$$
p_{T}^{q}>15 \mathrm{GeV}, \quad\left|\eta^{q}\right|<3.5, \quad \Delta R>0.4
$$


Here, we have assumed a perfect detector that can precisely measure the four-momenta of the final state partons and require the separation in $\Delta R\left(\equiv \sqrt{(\Delta \phi)^{2}+(\Delta \eta)^{2}}\right)$ between any two observable final state partons (not including neutrinos) to be larger than 0.4 , where $\Delta \phi$ and $\Delta \eta$ are the separation in azimuthal angle and rapidity, respectively. (We shall comment on the effect due to the finite resolution of the detector in the later part of this section.) Furthermore, in the $W g$ event, the additional $q^{\prime}$ jet (preferably in the forward direction) is required to escape detection, i.e., either its transverse momentum is less than $10 \mathrm{GeV}$ or its rapidity (in magnitude) is larger than 3.5. Similarly, the charged lepton $\ell^{-}\left(=e^{-}, \mu^{-}, \tau^{-}\right)$ from the decay of $\bar{t}$ in the $t \bar{t}$ event is required to be undetected, i.e., $p_{T}^{\ell^{-}}<10 \mathrm{GeV}$ or $\left|\eta^{\ell^{-}}\right|>$3.0. For clarity, we shall only include the positively charged state (i.e., $\pi^{+}$) in the following discussion. The inclusion of the opposite charged state (i.e., $\pi^{-}$) and other decay modes of $\tau^{+}$will be discussed in Sec. III D. The numbers of the signal and background events after imposing the above cuts are summarized in the second column of Table III in which the $b$-tagging efficiency (50\%, for tagging both $b$ and $\bar{b}$ jets) is included. The last three rows in Table 11 show the ratio of signal $(S)$ to background $(B)$ event number, the statistical significance of the signal, and the statistical uncertainty in the measured signal event rate.

At this stage of the analysis, the background rate is an order of magnitude larger than the signal rate. Moreover, the dominant background comes from the $W b \bar{b}$ process, followed by the $s$-channel single-top $(t \bar{b})$ process, the $t$-channel single-top $(W g)$ process and the $t \bar{t}$ pair process. To detect the signal event, further kinematic cuts are needed. In order to study the efficient cuts that can largely suppress the background rates while keeping most of the signal rates, we examine the distributions of the missing transverse energy $\left(E_{T}\right)$, transverse momentum of $b$ jet $\left(p_{T}^{b}\right)$, transverse momentum of $\pi^{+}\left(p_{T}^{\pi}\right)$, and the invariant mass of $b \bar{b}$ pair $\left(m_{b \bar{b}}\right)$. Their distributions are shown in Fig. 3, (The vertical axis shows the number of events per bin for all the figures presented in this section.)

b. Missing $E_{T}$ : As shown in Fig. 3, the typical size of the missing transverse energy in the signal event is larger than that in the $W b \bar{b}, t \bar{b}$ and $W g$ background events. This is because the main source of the missing energy in the signal and background events comes from the neutrino in the decay of $H^{+}$and $W^{+}$, respectively, and the mass of $H^{+}$considered 
TABLE III: Numbers of $A H^{+}$signal and background events for Case A, with $M_{A}=101 \mathrm{GeV}$ in the $b \bar{b} \pi^{+} E_{T}$ channel, at the LHC with an integrated luminosity of $100 \mathrm{fb}^{-1}$. The $b$-tagging efficiency (50\%, for tagging both $b$ and $\bar{b}$ jets) is included, and the kinematic cuts listed in each column are applied sequentially.

\begin{tabular}{c|c|c|c|c|c}
\hline \hline & Basic Cuts & $E_{T}>50 \mathrm{GeV}$ & $p_{T}^{\pi}>40 \mathrm{GeV}$ & $90<M_{b \bar{b}}<110 \mathrm{GeV}$ & With smearing \\
\hline$A H^{+}$ & 507 & 391 & 241 & 216 & 202 \\
\hline$H H^{+}$ & 48 & 38 & 24 & 0 & 4 \\
$W b \bar{b}$ & 11555 & 3111 & 864 & 67 & 62 \\
$t \bar{b}$ & 1228 & 614 & 163 & 12 & 11 \\
$W g$ & 567 & 236 & 68 & 11 & 9 \\
$t \bar{t}$ & 110 & 80 & 17 & 2 & 2 \\
\hline Signal $(S)$ & 507 & 391 & 241 & 216 & 202 \\
Bckg $(B)$ & 13507 & 4078 & 1135 & 92 & 2.32 \\
\hline$S / B$ & 0.038 & 0.095 & 0.212 & 2.35 & 21.6 \\
$S / \sqrt{B}$ & 4.36 & 6.12 & 7.14 & 22.5 & 0.08 \\
\hline$\sqrt{S+B} / S$ & 0.23 & 0.17 & 0.15 & 0.08 & 7 \\
\hline \hline
\end{tabular}

here is larger than the mass of the $W^{+}$boson. The average value of $\mathbb{E}_{T}$ in the $t \bar{t}$ event is larger than the other background processes, for the undetected charged lepton $\ell^{-}$from the decay of $\bar{t}$ also contributes to the missing energy of the event. The same argument also explains why the average missing energy in the $W g$ event (with an extra $q^{\prime}$ parton jet) is slightly larger than that in the $W b \bar{b}$ and $t \bar{b}$ events. In Table III, we show the number of signal and background events after demanding the missing energy of the event to be larger than $50 \mathrm{GeV}$. This cut increases the signal-to-background ratio by about a factor of 2.5 while keeping about $77 \%$ of the signal rate. The biggest reduction in the background rate comes from the $W b \bar{b}$ event (reduced by a factor of 3.7).

c. Transverse momentum of the $\pi$-jet: Another big difference between the signal and the background event signature is in the distribution of the transverse momentum of $\pi^{+}$ originated from the decay of $\tau^{+}$, cf. Fig. 3. It can be understood as follows. In the 
TABLE IV: Numbers of $H H^{+}$signal and background events for Case A, with $M_{H}=113 \mathrm{GeV}$ in the $b \bar{b} \pi^{+} E_{T}$ channel, at the LHC with an integrated luminosity of $100 \mathrm{fb}^{-1}$. The $b$-tagging efficiency (50\%, for tagging both $b$ and $\bar{b}$ jets) is included, and the kinematic cuts listed in each column are applied sequentially.

\begin{tabular}{c|c|c|c|c|c}
\hline \hline & Basic Cuts & $E_{T}>50 \mathrm{GeV}$ & $p_{T}^{\pi}>40 \mathrm{GeV}$ & $105<M_{b \bar{b}}<125 \mathrm{GeV}$ & With smearing \\
\hline$H H^{+}$ & 48 & 38 & 24 & 24 & 22 \\
\hline$A H^{+}$ & 507 & 391 & 241 & 26 & 43 \\
$W b \bar{b}$ & 11555 & 3111 & 864 & 58 & 54 \\
$t \bar{b}$ & 1228 & 614 & 163 & 11 & 11 \\
$W g$ & 567 & 236 & 68 & 13 & 12 \\
$t \bar{t}$ & 110 & 80 & 17 & 2 & 2 \\
\hline Signal $(S)$ & 48 & 38 & 24 & 24 & 120 \\
Bckg $(B)$ & 13966 & 4431 & 1352 & 110 & 0.19 \\
\hline$S / B$ & 0.003 & 0.008 & 0.018 & 0.22 & 2.03 \\
$S / \sqrt{B}$ & 0.41 & 0.57 & 0.65 & 2.26 & 0.53 \\
$\sqrt{S+B} / S$ & 2.47 & 1.76 & 1.55 & 0.49 & 22 \\
\hline \hline
\end{tabular}

signal event, the coupling of $H^{-} \tau^{+} \nu_{\tau}$ is almost left-handed due to the enhancement from large $\tan \beta$. Consequently, the $\tau^{+}$from $H^{+}$decay is produced preferentially left-handedly polarized, cf. Fig. 4. On the contrary, in the background events, $\tau^{+}$comes from the decay of the weak gauge boson $W^{+}$via left-handed weak current. In the dominant background $(W b \bar{b})$ event, the $W^{+}$boson is predominantly left-handed polarized. Therefore, $\tau^{+}$, as an anti-fermion, is right-handedly polarized when ignoring the small mass of $\tau^{+}$. In the weak decay of $\tau^{+} \rightarrow \pi^{+} \bar{\nu}_{\tau}$, the left-handed weak current forces $\bar{\nu}_{\tau}$ to be purely right-handed in the limit that the neutrino mass is ignored. Hence, to preserve angular momentum conservation, $\pi^{+}$would prefer moving along the direction of a left-handed $\tau^{+}$, and against the direction of a right-handed $\tau^{+}$, cf. Fig. 4. Namely, the angular distribution of $\pi^{+}$is $\left(1+\cos \theta_{\pi}\right)$ in the rest frame of a left-handed $\tau^{+}$, and $\left(1-\cos \theta_{\pi}\right)$ for a right-handed, where $\theta_{\pi}$ is the polar angle of $\pi^{+}$momentum with respect to the moving direction of $\tau^{+}$in the rest frame of $H^{+}$ 

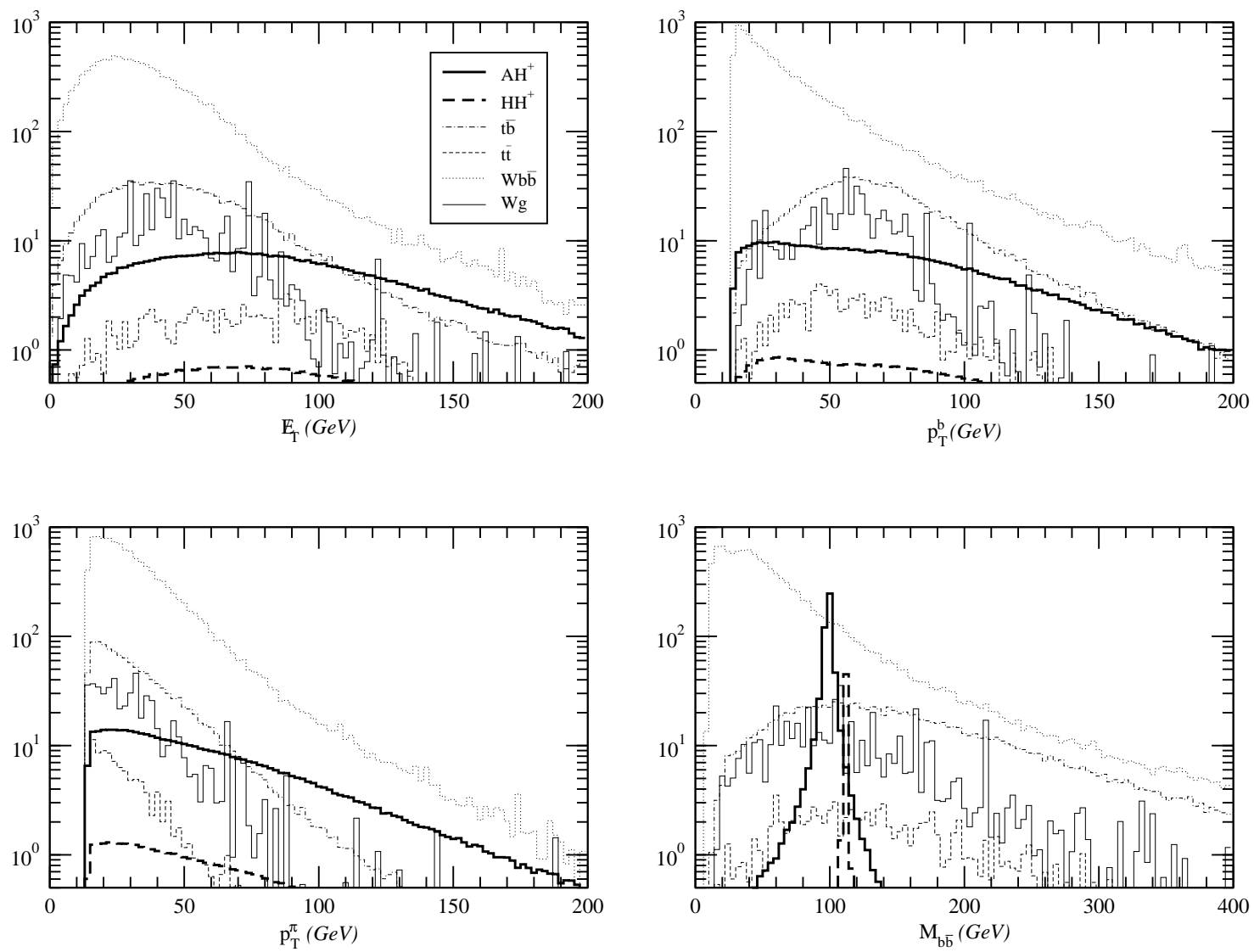

FIG. 3: Distributions of $\mathbb{E}_{T}, p_{T}^{b}, p_{T}^{\pi}$ and $m_{b \bar{b}}$ at the LHC for Case A, with $M_{A}=101 \mathrm{GeV}$ and $M_{H}=113 \mathrm{GeV}$ in the $b \bar{b} \pi^{+} \not_{T}$ channel, after imposing the basic cuts specified in Eq. (17).

(or $\left.W^{+}\right)$. Hence, the transverse momentum of $\pi^{+}$in $H^{+} \rightarrow \nu_{\tau} \tau^{+}\left(\rightarrow \pi^{+} \bar{\nu}_{\tau}\right)$ is typically larger than that in $W^{+} \rightarrow \nu_{\tau} \tau^{+}\left(\rightarrow \pi^{+} \bar{\nu}_{\tau}\right)$. To further suppress the background rate, we require $p_{T}^{\pi}>40 \mathrm{GeV}$. This cut increases the signal-to-background ratio by a factor of 2.2 at the cost of the reduction in signal rate by $40 \%$, while the background rate is reduced by a factor of 3.6. As clearly indicated in Table III, a similar reduction factor applies to all different background processes.

$d$. Invariant mass of the $b \bar{b}$-jet pair: At this stage of the analysis, the dominant background rate still comes from the $W b \bar{b}$ process whose rate is about 3.6 times the $A H^{+}$signal rate. Since in the $W b \bar{b}$ process, the $b \bar{b}$ pair originates from a virtual gluon conversion, the invariant mass of $b \bar{b}$ pair $\left(m_{b \bar{b}}\right)$ is generally small. This is clearly illustrated in Fig. 3. A similar, though less dramatic, result after imposing the kinematic cuts $\mathbb{E}_{T}>50 \mathrm{GeV}$ and 

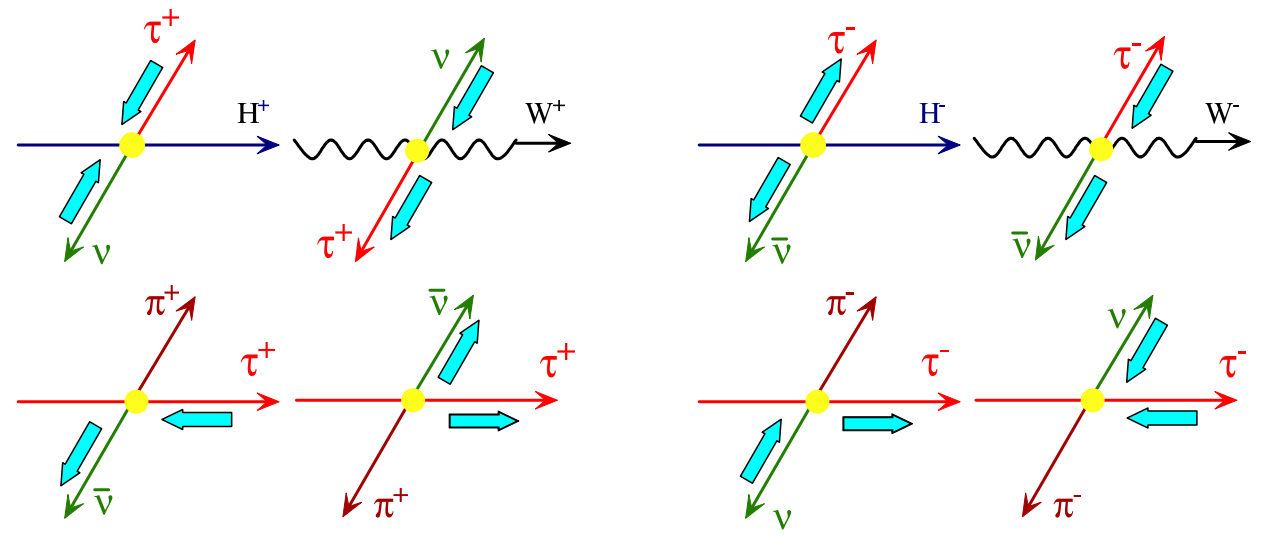

FIG. 4: $\tau^{+}$is left-handedly polarized in $H^{+} \rightarrow \tau^{+} \nu$, and right-handedly polarized in $W^{+} \rightarrow \tau^{+} \nu$. Moreover, $\pi^{+}$momentum depends on the polarization of $\tau^{+}$. (The thin arrow represents the moving direction, and the bold arrow represents the spin direction of the particle.) Similar plots for $\tau^{-}$are also shown in the right four diagrams.

$p_{T}^{\pi}>40 \mathrm{GeV}$, is also shown in Fig. 5 for comparison. To further improve the signal-tobackground ratio, we require the invariant mass of $b \bar{b}$ pair to be within $M_{A} \pm \sigma$, where $\sigma=10 \mathrm{GeV}$ is the expected experimental resolution for a $100 \mathrm{GeV}$ Higgs boson decaying into a $b \bar{b}$ pair [34]. As shown in Table III, the $W b \bar{b}$ background rate is reduced by a factor of 13, and the other background rates are also reduced by an order of magnitude. This yields $S / B=2.35$ and $S / \sqrt{B}=22.5$, with a total of 216 signal events, $q+q^{\prime} \rightarrow A(\rightarrow b \bar{b}) H^{+}\left(\rightarrow \nu \tau^{+}\left(\rightarrow \pi^{+} \bar{\nu}\right)\right)$, produced at the LHC with an integrated luminosity of $100 \mathrm{fb}^{-1}$.

Up to now, we have focused our discussion on the $A H^{+}$signal channel. A similar analysis can also be performed for the $\mathrm{HH}^{+}$signal channel, whose result is shown in Table IV where we have required the invariant mass of $b \bar{b}$ pair to be within $M_{H} \pm \sigma$ with $\sigma=10 \mathrm{GeV}$. As shown in Table III since the coupling of $W^{ \pm} H H^{\mp}$ is suppressed by a factor of $\sin (\beta-\alpha)$ as compared to that of $W^{ \pm} A H^{\mp}$ and $M_{H}>M_{A}$, the production rate of $H^{+}$is expected to be smaller than the $A H^{+}$rate by more than a factor of $\sin ^{2}(\beta-\alpha)$. For $M_{A}=101 \mathrm{GeV}$ and $\tan \beta=40$, this reduction rate is about 11 . Consequently, this $H H^{+}$signal is difficult to detect, for the background rate is still larger by a factor of 5 even after requiring $\#_{T}>50 \mathrm{GeV}$ and $p_{T}^{\pi}>40 \mathrm{GeV}$, cf. Table $\amalg$ and Fig. 5 . 


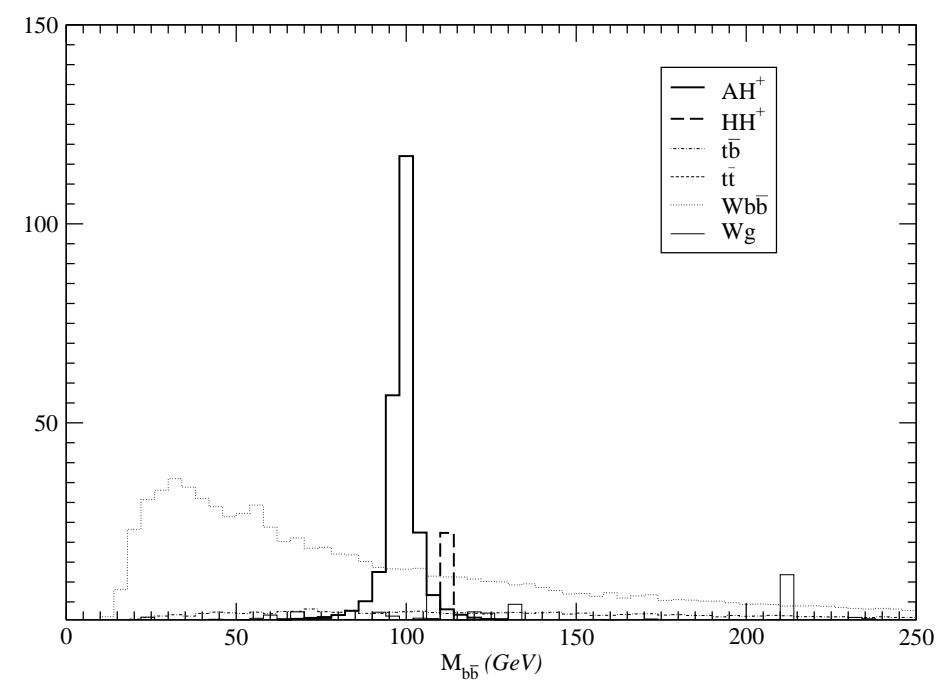

FIG. 5: Distributions of $m_{b \bar{b}}$ for Case A, with $M_{A}=101 \mathrm{GeV}$ and $M_{H}=113 \mathrm{GeV}$ in the $b \bar{b} \pi^{+} E_{T}$ channel, after imposing the additional cuts: $\not_{T}>50 \mathrm{GeV}$ and $p_{T}^{\pi}>40 \mathrm{GeV}$.

We therefore conclude that for a small $M_{A}$, the mass of $A$ can be determined from the $M_{b \bar{b}}$ distribution, as shown in Fig. 5. However, to measure the mass of $H$ via this channel is challenging.

e. Transverse mass of the charged Higgs Boson: As discussed above, the mass of $A$ can be determined from the invariant mass distribution of the $b \bar{b}$ pair. In order to test the mass relation, Eq. (11), predicted by the MSSM, the mass of $H^{+}$should also be measured. In the signal event, the decay product of the charged Higgs boson $\mathrm{H}^{+}$contains the charged pion $\pi^{+}$and the two (anti-)neutrinos $\left(\nu_{\tau}\right.$ and $\left.\bar{\nu}_{\tau}\right)$, which contribute to the missing transverse energy $\mathbb{H}_{T}$. Hence, we can examine the transverse mass of $H^{+}$defined as

$$
m_{T}=\sqrt{2 p_{T}^{\pi} E_{T}(1-\cos \phi)}
$$

where $\phi$ is the azimuthal angle between the $\pi^{+}$-jet and the missing transverse energy $\mathbb{F}_{T}$. It is expected that the shape of the $m_{T}$ distribution should exhibit a Jacobian peak behavior when the transverse momentum of $\pi^{+}$is large, for the dominant missing energy comes from $\nu_{\tau}$ in $H^{+} \rightarrow \nu_{\tau} \tau^{+}\left(\rightarrow \pi^{+} \bar{\nu}_{\tau}\right)$. This feature is clearly illustrated in Fig. 6. The signal event shows a Jacobian peak around $M_{H^{+}}(126 \mathrm{GeV})$, while the dominant $W b \bar{b}$ background event shows a Jacobian peak around $m_{W}(80.2 \mathrm{GeV})$ but with a long tail into the larger $M_{b \bar{b}}$ region. 


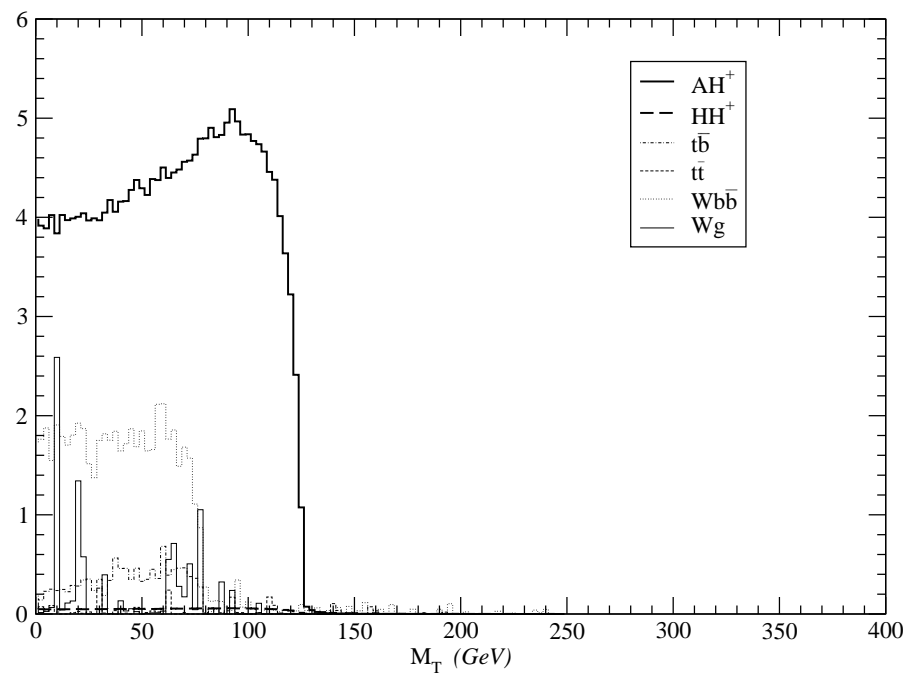

FIG. 6: Distributions of the transverse mass $m_{T}$, defined in Eq. (18), for Case A, after imposing the additional cut: $\left|m_{b \bar{b}}-m_{A / H}\right|<10 \mathrm{GeV}$.

The long tail comes from the $W b \bar{b}$ events in which the transverse momentum of the $W^{+}$ boson is large. Since the other background event rates are much smaller than the $\mathrm{AH}^{+}$ signal rate, we conclude that the charged Higgs boson mass can be accurately extracted from the transverse mass distribution.

f. Detector Effects: In reality, the performance of the detector is not perfect. To study the effect due to the finite detection efficiency of the detector, we repeat the above Monte Carlo analysis after smearing all the final state parton momenta by a Gaussian distribution with

$$
\frac{\Delta E}{E}=\frac{50 \%}{\sqrt{E}}
$$

where $E$ is the energy of the observed parton and the resolution of the energy measurement is assumed to be $50 \% \sqrt{E}$. The distributions of invariant mass $m_{b \bar{b}}$ and the transverse mass $m_{T}$ become slightly broader, as shown in Fig. 17. However, both the signal and background rates, as shown in the last column of Tables II and IV are almost the same as those obtained with a perfect detector.

Finally, we note that in the above analysis we have limited ourselves to a very minimal set of kinematic cuts to enhance the signal-to-background ratio (by about a factor of 60) while keeping most of the signal events (about half of them). There are other distributions 

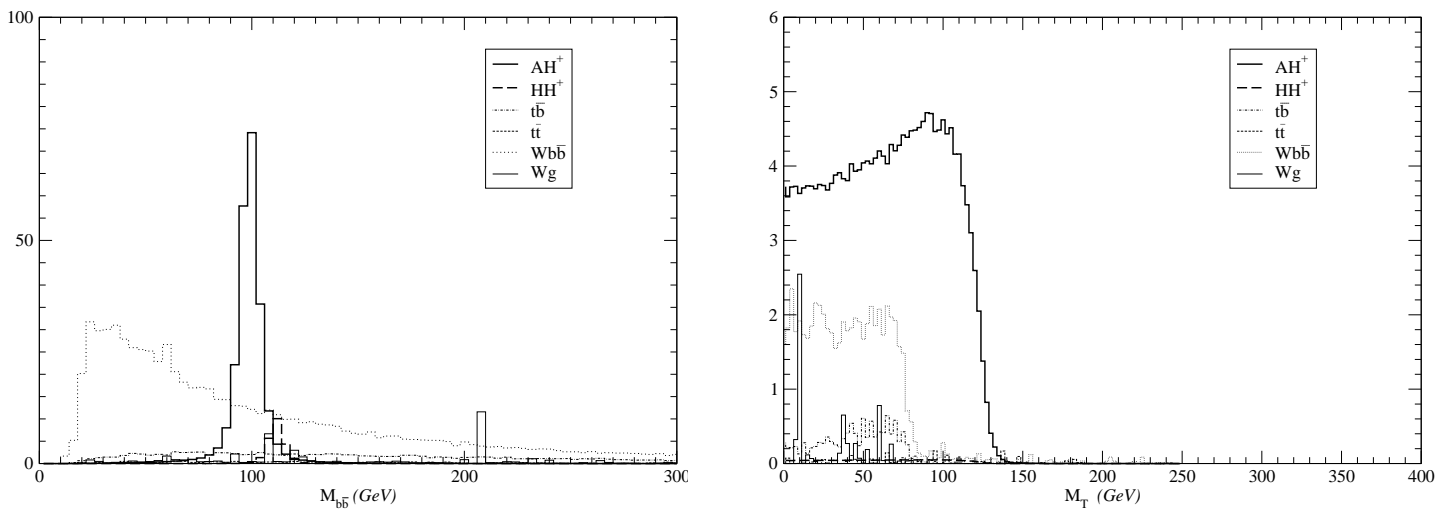

FIG. 7: Distributions of invariant mass $m_{b \bar{b}}$ (after imposing the basic cuts, $E_{T}$ and $p_{T}^{\pi}$ cuts) and transverse mass $m_{T}$ (after imposing all the kinematic cuts) for Case A, with $M_{A}=101 \mathrm{GeV}$ and $M_{H}=113 \mathrm{GeV}$, after smearing the observable parton momenta by $50 \% \sqrt{E}$.

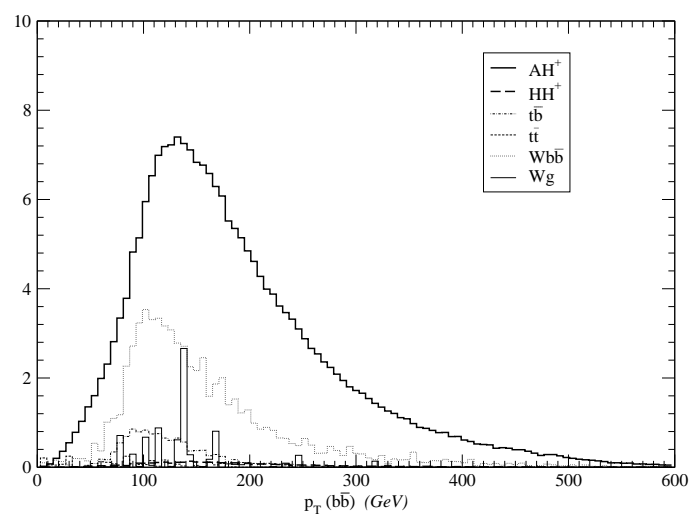

FIG. 8: Transverse momentum distribution of the $b \bar{b}$ pair for Case A, after imposing the additional cut: $\left|m_{b \bar{b}}-m_{A / H}\right|<10 \mathrm{GeV}$.

that can further distinguish signal from background events. For example, as shown in Fig. 8. the transverse momentum of the $b \bar{b}$ pair in the $A H^{+}$signal events is typically larger than that in the background events, even after imposing the kinematic cuts $\mathbb{E}_{T}>50 \mathrm{GeV}$ and $p_{T}^{\pi}>40 \mathrm{GeV}$. This is because the scattering amplitude of the $A H^{+}$signal event is a p-wave amplitude, cf. Eq. (3), and $M_{H^{+}}$is larger than $M_{A}$, so that $A$ is boosted to produce a large transverse momentum which is typically about $100 \mathrm{GeV}$ in this case. 


\section{B. $M_{A}=166 \mathrm{GeV}$}

We shall extend our analysis to the production of a charged Higgs boson with its mass only slightly more than the top quark mass $(174 \mathrm{GeV})$ so that the dominant decay mode of $H^{+}$remains to be the $\tau^{+} \nu$ channel. We take $M_{A}=166 \mathrm{GeV}, \tan \beta=40$, and all the other SUSY parameters (soft-breaking masses and $\mu$ parameter) to be $500 \mathrm{GeV}$. Consequently, the masses of the other Higgs bosons are calculated from HDECAY [32], which gives $M_{h}=$ $112 \mathrm{GeV}, M_{H}=163 \mathrm{GeV}$ and $M_{H^{+}}=182 \mathrm{GeV}$. The relevant decay branching ratios are $B(A \rightarrow b \bar{b})=0.90, B(H \rightarrow b \bar{b})=0.90, B\left(H^{+} \rightarrow \tau^{+} \nu\right)=0.90$, and $B\left(\tau^{+} \rightarrow \pi^{+} \nu\right)=0.11$. The relevant total decay widths are $\Gamma_{A}=5.6 \mathrm{GeV}, \Gamma_{H}=5.5 \mathrm{GeV}$ and $\Gamma_{H^{ \pm}}=0.68 \mathrm{GeV}$, respectively. They are summarized in Table II for a quick reference. In this case, $M_{H}$ is about the same as $M_{A}$, and $\sin (\beta-\alpha) \sim 1$, hence, the production rates of $A H^{+}$and $H H^{+}$ are about the same. Since the decay branching ratios of $A \rightarrow b \bar{b}$ and $H \rightarrow b \bar{b}$ are also similar, we shall include both the $\mathrm{AH}^{+}$and $\mathrm{HH}^{+}$event rates when we compute the signal rate in this case. Following the same analysis procedure as that for the small $M_{A}$ case, we show the numbers of signal and background events in Table $\mathrm{V}$. After imposing all the kinematic cuts, the signal-to-background ratio increases by two orders of magnitude at the cost of half of signal events, and there are about 140 signal events and 50 background events. In Fig. 9, we show the distributions of the invariant mass $m_{b \bar{b}}$ (after imposing the basic cuts, $\not_{T}$ and $p_{T}^{\pi}$ cuts) and transverse mass $m_{T}$ (after imposing all the kinematic cuts), which are obtained after smearing the observable parton momenta by $50 \% \sqrt{E}$ to mimic the effect of the finite resolution of the detector.

\section{C. $M_{A}=250 \mathrm{GeV}$}

When the mass of the $\mathrm{H}^{+}$is larger than the sum of the masses of top and bottom quarks but not too large that the supersymmetric decay modes become significant, the dominant decay mode of $H^{+}$is likely to be $H^{+} \rightarrow t \bar{b}$. Here, we study how to detect the signal event in this decay mode.

To simplify the discussion, we shall concentrate on the semi-leptonic decay mode of top quark, and the $b \bar{b}$ decay mode of $A$ or $H$. Hence, the signal events considered here are 
TABLE V: Numbers of $A H^{+}$and $H H^{+}$signal and background events for Case B, with $M_{A}=$ $166 \mathrm{GeV}$ and $M_{H}=163 \mathrm{GeV}$ in the $b \bar{b} \pi^{+} E_{T}$ channel, at the LHC with an integrated luminosity of $100 \mathrm{fb}^{-1}$. The $b$-tagging efficiency (50\%, for tagging both $b$ and $\bar{b}$ jets) is included, and the kinematic cuts listed in each column are applied sequentially.

\begin{tabular}{c|c|c|c|c|c}
\hline & Basic Cuts & $H_{T}>50 \mathrm{GeV}$ & $p_{T}^{\pi}>40 \mathrm{GeV}$ & $155<M_{b \bar{b}}<175 \mathrm{GeV}$ & With smearing \\
\hline$A H^{+}$ & 126 & 111 & 85 & 71 & 65 \\
$H H^{+}$ & 129 & 114 & 87 & 72 & 66 \\
\hline$W b \bar{b}$ & 11560 & 3102 & 840 & 33 & 34 \\
$t \bar{b}$ & 1221 & 607 & 164 & 10 & 10 \\
$W g$ & 783 & 318 & 11 & 5 & 6 \\
$t \bar{t}$ & 108 & 79 & 18 & 1 & 131 \\
\hline Signal $(S)$ & 255 & 225 & 171 & 143 & 50 \\
Bckg $(B)$ & 13672 & 4106 & 1031 & 48 & 2.6 \\
\hline$S / B$ & 0.02 & 0.05 & 0.17 & 3.0 & 18.6 \\
$S / \sqrt{B}$ & 2.18 & 3.51 & 5.33 & 20.8 & 0.10 \\
$\sqrt{S+B} / S$ & 0.46 & 0.29 & 0.20 & 0.09 & 13 \\
\hline
\end{tabular}

produced via

$$
\begin{aligned}
& q q^{\prime} \rightarrow A(\rightarrow b \bar{b}) H^{+}\left(\rightarrow \bar{b} t\left(\rightarrow b \ell^{+} \nu\right)\right), \\
& q q^{\prime} \rightarrow H(\rightarrow b \bar{b}) H^{+}\left(\rightarrow \bar{b} t\left(\rightarrow b \ell^{+} \nu\right)\right),
\end{aligned}
$$

where $\ell^{+}=e^{+}$or $\mu^{+}$. The signature of the signal event is four $b$-jets plus one isolated lepton and missing transverse energy. To detect the signal event, we require the four $b$-jets and the isolated charged lepton to be well separated in $\Delta R$ with large $p_{T}$ in the central rapidity region, in addition to a $\mathbb{E}_{T}$ signature. In the following, we assume that the efficiency for tagging all four $b$-jets is $25 \%$, which is included in the calculation of the event rates to be discussed below. The dominant SM background for such kind of signature comes from the $t \bar{t} b \bar{b}$ events, produced via

$$
q \bar{q}, g g \rightarrow b \bar{b} t\left(\rightarrow b \ell^{+} \nu\right) \bar{t}\left(\rightarrow \bar{b} W^{-}\right)
$$



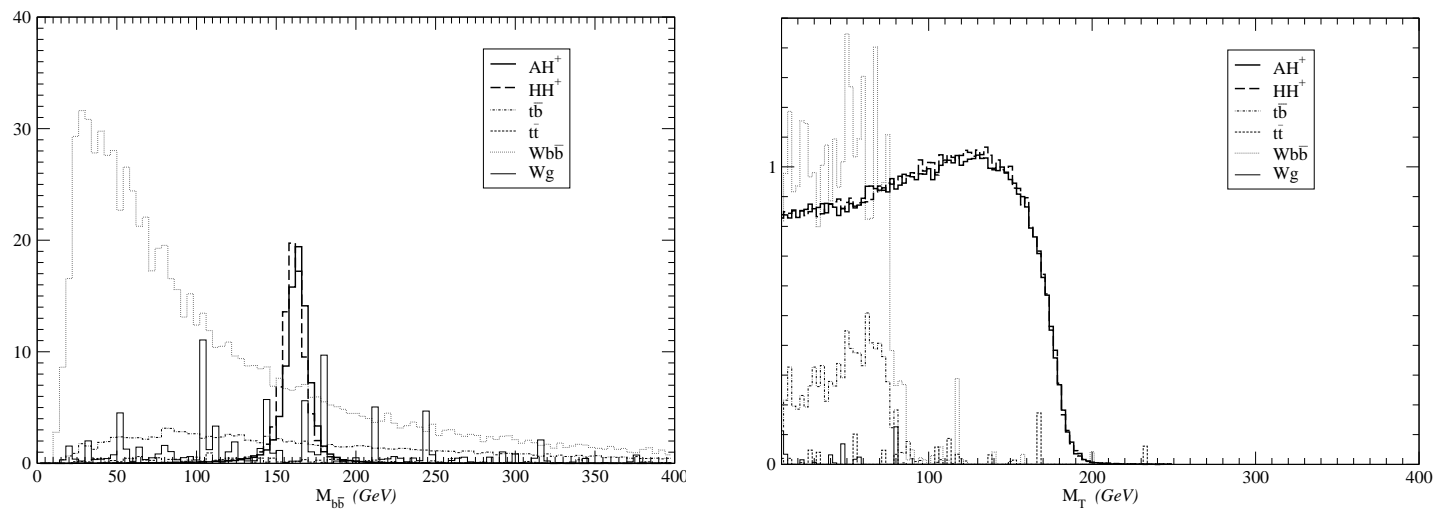

FIG. 9: Distributions of invariant mass $m_{b \bar{b}}$ (after imposing the basic cuts, $\mathbb{E}_{T}$ and $p_{T}^{\pi}$ cuts) and transverse mass $m_{T}$ (after imposing all the kinematic cuts) for Case B, with $M_{A}=166 \mathrm{GeV}$ and $M_{H}=163 \mathrm{GeV}$, after smearing the observable parton momenta by $50 \% \sqrt{E}$.

When the decay products of $W^{-}$escape detection, this background event will mimic the signal event signature. At the LHC, the $t \bar{t} b \bar{b}$ rate is dominated by the gluon fusion process due to its large parton luminosity. As shown in Table VI after imposing the following basic kinematic cuts: ${ }^{3}$

$$
\begin{aligned}
p_{T}^{\ell^{+}} & >15 \mathrm{GeV}, \quad\left|\eta^{\ell^{+}}\right|<3.0, \quad E_{T}>20 \mathrm{GeV} \\
p_{T}^{b} & >20 \mathrm{GeV}, \quad\left|\eta^{b}\right|<2.0 \\
\Delta R & >0.4
\end{aligned}
$$

the $g g \rightarrow t \bar{t} b \bar{b}$ rate is larger than the $q \bar{q} \rightarrow t \bar{t} b \bar{b}$ rate by two orders of magnitude. Here, we have required the separation in $\Delta R$ between any two final state partons among the four $b$-jets and the charged lepton $\ell^{+}$to be larger than 0.4 .

The signal rate depends on SUSY parameters. We take $M_{A}=250 \mathrm{GeV}, \tan \beta=40$, and all the other SUSY parameters (soft-breaking masses and $\mu$ parameter) to be $500 \mathrm{GeV}$. Consequently, $M_{H}=248 \mathrm{GeV}$ and $M_{H^{+}}=261 \mathrm{GeV}$. The relevant decay branching ratios are $B(A \rightarrow b \bar{b})=0.89, B(H \rightarrow b \bar{b})=0.89, B\left(H^{+} \rightarrow t \bar{b}\right)=0.79$, and $B\left(t \rightarrow b e^{+} \nu\right)=0.11$. The relevant total decay widths are $\Gamma_{A}=7.9 \mathrm{GeV}, \Gamma_{H}=7.8 \mathrm{GeV}$ and $\Gamma_{H^{ \pm}}=4.2 \mathrm{GeV}$, as

\footnotetext{
${ }^{3}$ Here, we impose a set of stronger cuts than those given in Eq. (17) in order to have a more reliable estimate of the $t \bar{t} b \bar{b}$ background rate.
} 
TABLE VI: Numbers of $A H^{+}$and $H H^{+}$signal and background events for Case C, with $M_{A}=$ $250 \mathrm{GeV}$ and $M_{H}=248 \mathrm{GeV}$ in the $b \bar{b} b \bar{b} \ell^{+} H_{T}$ channel, at the LHC with an integrated luminosity of $100 \mathrm{fb}^{-1}$. The $b$-tagging efficiency (25\%, for tagging all the four $b$ jets) is included, and the kinematic cuts listed in each column are applied sequentially.

\begin{tabular}{c|c|c}
\hline \hline & Basic Cuts & Veto Cuts \\
\hline$A H^{+}$ & 18 & 18 \\
$H H^{+}$ & 18 & 18 \\
\hline \hline$g g \rightarrow t \bar{t} b \bar{b}$ & 1632 & 3 \\
\hline$q q \rightarrow t \bar{t} b \bar{b}$ & 105 & 0 \\
\hline \hline Signal $(S)$ & 36 & 36 \\
Bckg $(B)$ & 1737 & 3 \\
\hline \hline
\end{tabular}

summarized in Table [I] For this set of parameters, the event rates of the $A H^{+}$and $H H^{+}$ modes are about the same due to the decoupling limit, i.e., $M_{A}$ becomes large. Furthermore, the total signal rate (the sum of the $\mathrm{AH}^{+}$and $\mathrm{HH}^{+}$rate) is about one hundredth of the $t \bar{t} b \bar{b}$ background rate, cf. Table $\overline{V I}$, after the basic cuts, and the sum of the $A H^{+}$and $H H^{+}$ signal rates yields about 36 events at the LHC. We have checked that our calculation of the $t \bar{t} b \bar{b}$ cross section agrees with that in Refs. [35, 36], where we have chosen the scale for evaluating parton distribution functions and strong coupling to be the invariant mass of the final state particles. (The $t \bar{t} b \bar{b}$ cross section can vary as much as $50 \%$ with a different choice of the scale [35].)

As noted above, the $t \bar{t} b \bar{b}$ background events contain an extra $W^{-}$boson in the final state, as compared to the signal event. To suppress this large background event, we need to veto the additional $W^{-}$boson which can decay into di-jets or a charged lepton plus neutrino. When $W^{-}$decays into the di-jet mode, there will be additional hadronic activities in the central rapidity region, hence, one needs to apply kinematic cuts to veto the additional high $p_{T}$ jets in the central rapidity region. When $W^{-}$decays into a charged lepton plus neutrino, an additional charged lepton is produced in the final state. Below, we first discuss the case that $W^{-}$decays into the leptonic mode with the charged lepton $\left(\ell^{-}\right)$being $e^{-}$ 
or $\mu^{-}$, which accounts for two ninth of the top quark decay branching ratio. We veto the additional charged lepton with a transverse momentum exceeding $10 \mathrm{GeV}$ and rapidity (in magnitude) less than 3.0. It turns out that the veto efficiency is so high (around 99\%) that the background rate is down by three orders of magnitude and yields 2 events of $\bar{t} \rightarrow \bar{b} W^{-}\left(\rightarrow \nu \ell^{-}\right)$with $\ell^{-}=e^{-}$or $\mu^{-}$. Consequently, the signal rate is about one order of magnitude larger than this background rate. Though the above analysis is adequate for $\ell^{-}=e^{-}$or $\mu^{-}$, it is less accurate for $\ell^{-}=\tau^{-}$when $\tau^{-}$decays further to either leptons or hadrons. (The decay branching ratio of $W^{-} \rightarrow \bar{\nu} \tau^{-}$is about one ninth.) Hence, we improve the calculation of the $\bar{t} \rightarrow \bar{b} W^{-}\left(\rightarrow \bar{\nu} \tau^{-}\right)$background rate by considering the leptonic decay mode $\tau^{-} \rightarrow \ell^{-} \nu_{\tau} \bar{\nu}$ (with $\ell^{-}=e^{-}$or $\mu^{-}$) and the hadronic decay modes of tau, such as $\tau^{-} \rightarrow \pi^{-} \nu_{\tau}$ or $\rho^{-} \nu_{\tau}$. For the $\tau^{-} \rightarrow \ell^{-} \nu_{\tau} \bar{\nu}$ decay mode, we veto events in which $p_{T}^{\ell^{-}}>10 \mathrm{GeV}$ and $\left|\eta^{\ell^{-}}\right|<3.0$. After taking into account the decay branching ratio of tau into the leptonic mode (about 35\%), this amounts to 1 event of $\bar{t} \rightarrow \bar{b} W^{-}\left(\rightarrow \bar{\nu} \tau^{-}\left(\rightarrow \ell^{-} \nu_{\tau} \bar{\nu}\right)\right)$ passing through the vetoing cut. It is straightforward to find out the background rate of $\bar{t} \rightarrow \bar{b} W^{-}\left(\rightarrow \bar{\nu} \tau^{-}\left(\rightarrow \pi^{-} \nu_{\tau}\right)\right)$, after vetoing the additional central jet (i.e., $\pi^{-}$jet) that has transverse momentum larger than $10 \mathrm{GeV}$ and rapidity (in magnitude) less than 3.5. We find that the vetoing efficiency for the $\tau^{-} \rightarrow \pi^{-} \nu_{\tau}$ event, whose decay branching ratio is about $11 \%$, is so high that its contribution to the $t \bar{t} b \bar{b}$ background rate is negligibly small. The similar conclusion also holds for the $\tau^{-} \rightarrow \nu_{\tau} \rho^{-}\left(\rightarrow \pi^{-} \pi^{0}\right)$ mode, whose decay branching ratio is about $25 \%$. For this decay mode, we first check whether the separation of $\pi^{-}$and $\pi^{0}$ in $\Delta R$ is within 0.4. If yes, we sum up their momenta to form one big jet and follow the same analysis as that for $\tau^{-} \rightarrow \pi^{-} \nu_{\tau}$. If not, we veto any additional central jet (i.e., $\pi^{-}$or $\pi^{0}$ jet) that has transverse momentum larger than $10 \mathrm{GeV}$ and rapidity (in magnitude) within 3.5. In order for the $\bar{t} \rightarrow \bar{b} W^{-}\left(\rightarrow \bar{q} q^{\prime}\right)$ background event to mimic the signal event, the decay products of $W^{-}$have to escape detection. When the separation of $\bar{q}$ and $q^{\prime}$ in $\Delta R$ is within 0.4, we sum up their momenta to form one big jet and veto the event when the transverse momentum of the big jet is larger than $10 \mathrm{GeV}$ and rapidity (in magnitude) smaller than 3.5. Otherwise, we veto any additional central jet (i.e., $\bar{q}$ or $q^{\prime}$ jet) that has transverse momentum larger than $10 \mathrm{GeV}$ and rapidity (in magnitude) within 3.5. This amounts to a negligibly small number of $\bar{t} \rightarrow \bar{b} W^{-}\left(\rightarrow \bar{q} q^{\prime}\right)$ events at the LHC, for its vetoing efficiency 
is close to $100 \%$. In Table VI, we sum up all the decay modes of $W^{-}$discussed above to estimate the background rate $t \bar{t} b \bar{b} \rightarrow b \bar{b} b \bar{b} \ell^{+} \#_{T}$ which is about one order of magnitude smaller than the signal rate. Furthermore, the distribution of the transverse mass of $\ell^{+}$ and $E_{T}$, which is the transverse mass of the $W^{+}$boson in the signal event, can be used to further discriminate the signal from the background events. This is because the vetoing cuts generate additional missing energy in the background event, while in the signal event, the $\#_{T}$ is entirely generated by the neutrino from $W^{+}$boson decay. It is desirable to check the above estimate using a full event generator (including effects of parton showering and hadronization) combined with a realistic detector simulation, which is however beyond the scope of this paper.

Next, we illustrate how to reconstruct the mass of $A$ and $H^{+}$in the signal event $A(\rightarrow$ $b \bar{b}) H^{+}\left(\rightarrow \bar{b} t\left(\rightarrow b \ell^{+} \nu\right)\right)$. A similar method also applies to the $H H^{+}$signal event. Since both $\ell^{+}$and $\nu$ come from an on-shell $W^{+}$boson decay, we can use the $W$-boson mass constraint and $E_{T}$ information to specify the longitudinal momentum $\left(p_{z}(\nu)\right)$ of the neutrino. The above procedure leads to two possible solutions of $p_{z}(\nu)$. Sometimes, only one of the two solutions is kinematically allowed, but most of the time both of them are physical solutions for a signal event. Therefore, one has to fix a prescription [40] to choose the one which will most likely give the correct distribution of the invariant mass of $e^{+}, \nu_{e}$ and $b$. Since $W$-boson, top quark and $H^{+}$boson are all heavy particles, they and their decay products are likely to be produced in the central rapidity region. Hence, we choose the one with the smaller magnitude in $p_{z}(\nu)$ to reconstruct the $W^{+}$boson. In the signal event, there are four $b$-jets. (We consider the case that a $\bar{b}$-jet cannot be experimentally distinguished from a $b$-jet.) We pair the reconstructed $W^{+}$boson with any one of the four $b$-jets to calculate the invariant mass of $W^{+} b$ and choose the $b$-jet that yields a mass closest to the nominal top mass $(174 \mathrm{GeV})$ to be the one produced from the decay of $t$. In Fig. 10)(a), we show the distribution of the reconstructed top quark mass which peaks around $174 \mathrm{GeV}$ with a $17 \mathrm{GeV}$ half-of-maximum width. In order to pair the two correct $b$-jets, among the remaining three $b$-jets, to reconstruct the mass of the CP-odd Higgs boson $A$, we make use of the mass inequality implied by the MSSM mass relation, Eq. (11). Namely, the mass of $A$ is smaller than the mass of $H^{+}$. (As $M_{A}$ becomes large, the mass of $H$ is about the same as the mass 

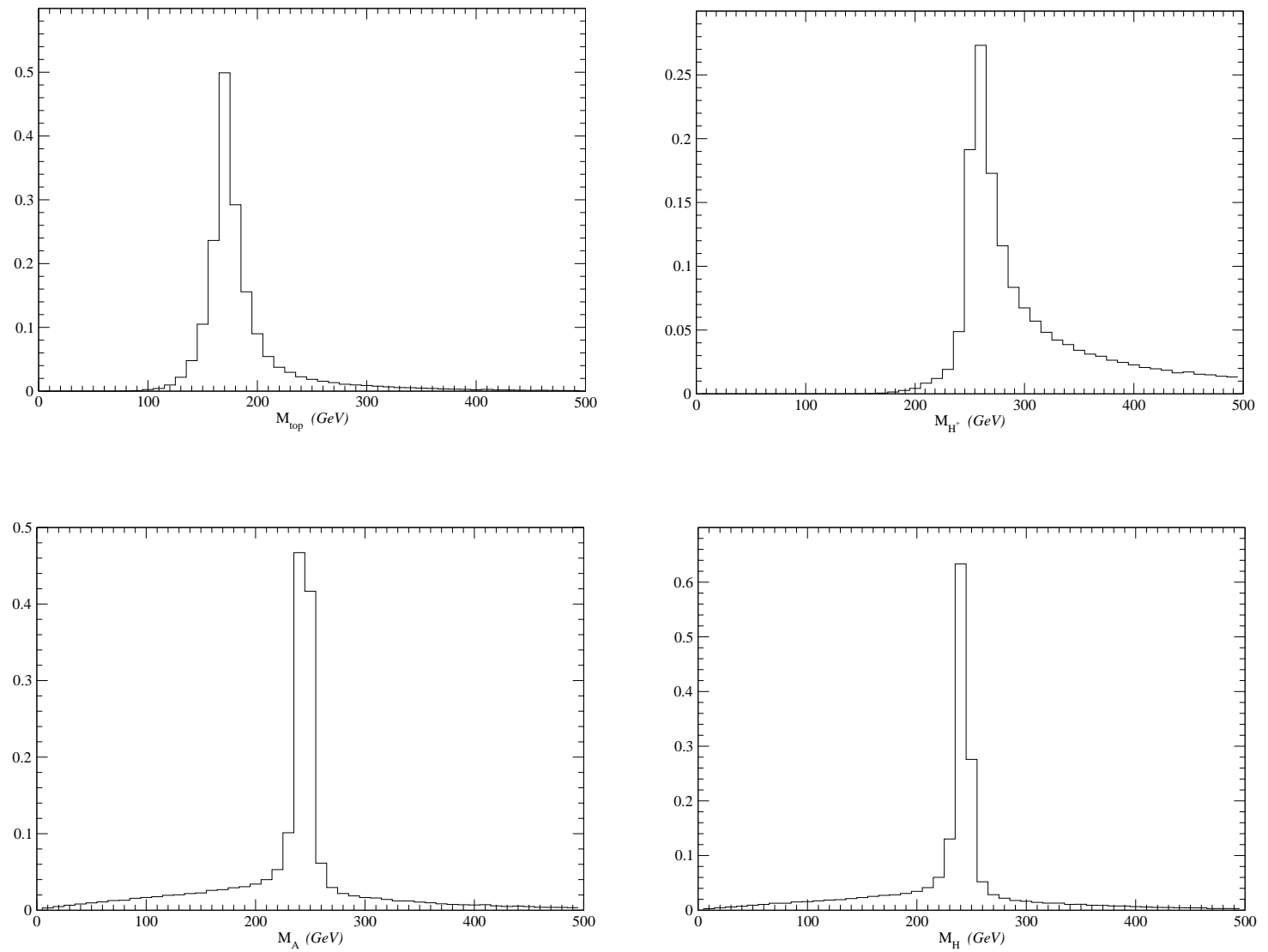

FIG. 10: The invariant mass distributions of the reconstructed (a) top quark $t$, (b) charged Higgs boson $H^{+}$, (c) CP-odd Higgs Boson $A$, and (d) CP-even heavy Higgs boson $H$, for Case C, with $M_{H^{+}}=261 \mathrm{GeV}, M_{A}=250 \mathrm{GeV}$ and $M_{H}=248 \mathrm{GeV}$, after imposing all the kinematic cuts discussed in the text.

of $A$.) We loop over the remaining three $b$-jets (labelled as $b_{1,2,3}$ ), and calculate the invariant mass of $t b_{1}$, denoted as $m_{t b_{1}}$, and the invariant mass of $b_{2} b_{3}$, denoted as $m_{b_{2} b_{3}}$. We choose the assignment of the three $b$-jets so that $m_{t b_{1}}>m_{b_{2} b_{3}}$ and the difference in $m_{t b_{1}}^{2}$ and $m_{b_{2} b_{3}}^{2}$ is closest to $M_{W}^{2}$. Following this procedure, we obtain the distributions of $M_{A}$ and $M_{H^{+}}$which are shown in Figs. 10(b) and 10(c). The distribution of the reconstructed $M_{A}$ peaks around $250 \mathrm{GeV}$ with a $10 \mathrm{GeV}$, half-of-maximum width, and the reconstructed $M_{H^{+}}$peaks around $261 \mathrm{GeV}$ with a $20 \mathrm{GeV}$ half-of-maximum width. The broad width in the reconstructed $M_{H^{+}}$ distribution is caused by the wrong assignment of the $b$-jets, for the difference in $M_{H^{+}}$and $M_{A}$ (about $13 \mathrm{GeV}$ ) is at the same order as the decay widths of $A$ and $H^{+}$. Hence, even with 
the crude analysis discussed above, it is possible to reconstruct the mass of $A$ and $H^{+}$in the $A H^{+} \rightarrow b \overline{b b} t$ signal event. Similarly, $M_{H}$ can also be reconstructed for the $H H^{+} \rightarrow b \overline{b b} t$ signal event, whose result is shown in Fig. 10(d). A more complicated analysis using the likelihood method, such as the neural network analysis, would certainly improve the above conclusion.

Finally, we comment on the effect due to the finite resolution of the detector. Again, we smear the four-momenta of the final state partons (except neutrino) by assuming a Gaussian error of $50 \% \sqrt{E}$, where $E$ is the energy of the parton. Repeating the above analysis, we obtain the distributions of the reconstructed $M_{t}, M_{A}$ (or $M_{H}$ ) and $M_{H^{+}}$as shown in Fig. [1]. Except the small difference in the height of the peak, they are similar to those obtained for a perfect detector, as shown in Fig. 10.

\section{Discussions}

Before concluding this section, we would like to summarize the results of our Monte Carlo analysis and discuss the need for a realistic simulation of the detector and a full event generator analysis. In this work, we considered three different signal scenarios as given in Table III. In Case A and Case B, the dominant decay mode of $H^{+}$is $H^{+} \rightarrow \tau^{+} \nu_{\tau}$. For Case A, the SUSY parameters of the Higgs sector are in the mixing regime $[\sin (\beta-\alpha) \sim 0.3-0.8]$, so that $M_{H}$ differs from $M_{A}$. For Case B, the SUSY parameters of the Higgs sector are in the decoupling regime $[\sin (\beta-\alpha) \sim 1]$, so that $M_{H}$ is almost the same as $M_{A}$. In Case $\mathrm{C}$, the $t \bar{b}$ mode opens and becomes the dominant decay channel of $\mathrm{H}^{+}$, so that it provides a different event signature. The results of our Monte Carlo analysis are separately summarized as follows.

To detect the signal events for Case $\mathrm{A}$ and Case $\mathrm{B}$, we consider the decay modes of $A \rightarrow b \bar{b}$ (or $H \rightarrow b \bar{b}$ ) and $H^{+} \rightarrow \tau^{+} \nu_{\tau}$ with $\tau^{+} \rightarrow \pi^{+} \bar{\nu}_{\tau}$ in the $A H^{+}$(or $H H^{+}$) events. The relevant decay widths and branching ratios are summarized in Table II for a quick reference. The dominant SM background events for these cases come from $W b \bar{b}, t \bar{b}, W g$, and $t \bar{t}$ events, as defined in Eq. (16).

Firstly, we discuss Case A, in which the production rate of $\mathrm{HH}^{+}$is smaller than $\mathrm{AH}^{+}$ by a factor of $\sin ^{2}(\beta-\alpha) \sim 0.11$. After imposing the basic cuts, specified in Eq. (17), 

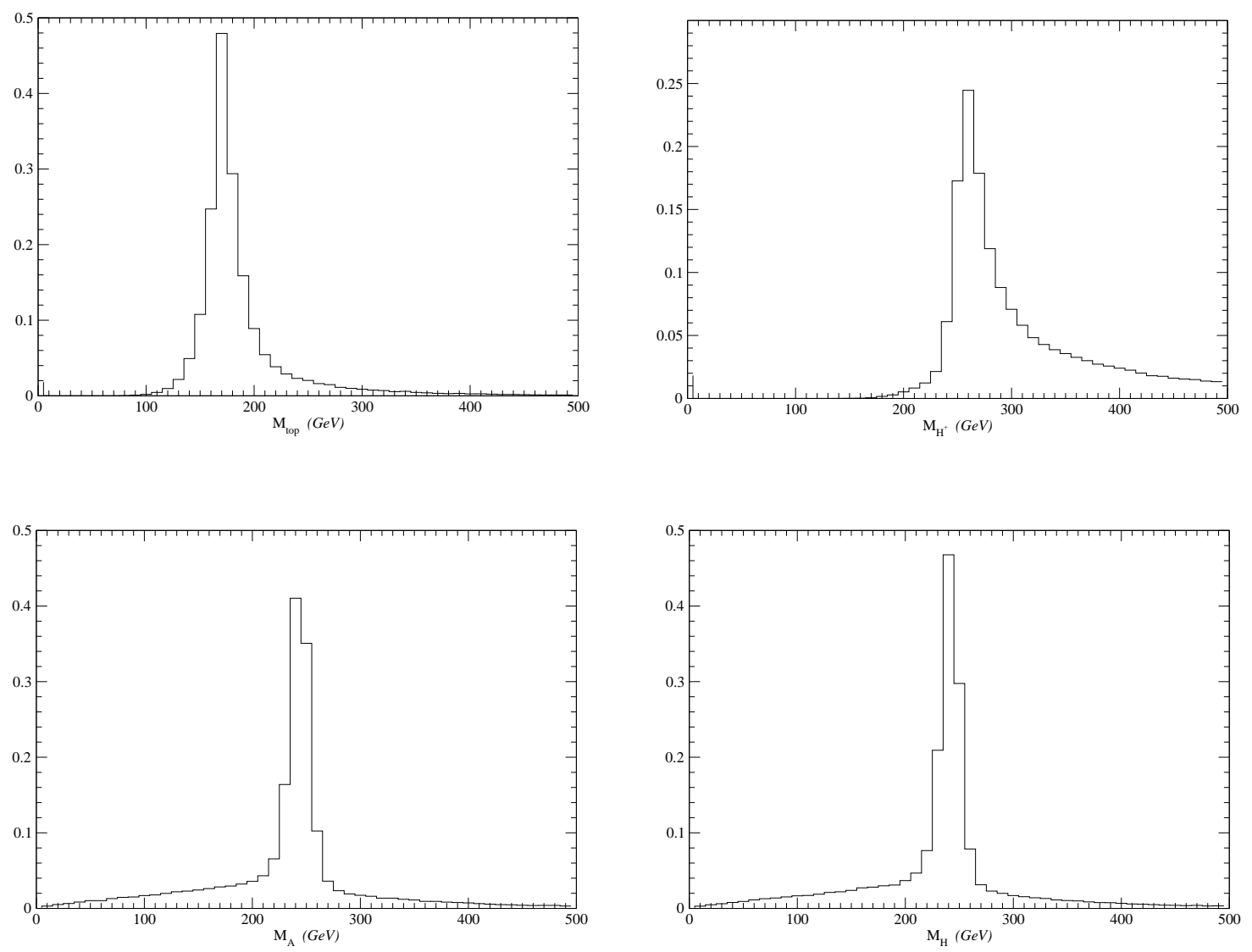

FIG. 11: The invariant mass distributions of the reconstructed (a) top quark $t$, (b) charged Higgs boson $H^{+}$, (c) CP-odd Higgs Boson $A$, and (d) CP-even heavy Higgs boson $H$, for Case C, with $M_{H^{+}}=261 \mathrm{GeV}, M_{A}=250 \mathrm{GeV}$ and $M_{H}=248 \mathrm{GeV}$, after smearing the observable parton momenta by $50 \% \sqrt{E}$ and imposing all the kinematic cuts discussed in the text.

and assuming a $50 \%$ of $b$-tagging efficiency for tagging both $b$-jets in the event, we obtain the signal and background event rates as shown in the second column of Table III for the LHC with an integrated luminosity of $100 \mathrm{fb}^{-1}$. By noting that the background events (particularly the $W b \bar{b}$ events) tend to have smaller missing energy, we impose a cut, $\not_{T}>$ $50 \mathrm{GeV}$, to suppress various background rates. The results are shown in the third column of Table III. Furthermore, the polarization of $\tau^{+}$in the signal events (left-handedly polarized) is different from that in the SM background events (right-handedly polarized). Consequently, the typical transverse momentum of $\pi^{+}$from the decay of $\tau^{+}$is higher in the signal events than in the SM background events, cf. Fig. 4. Hence, we impose another cut, $p_{T}^{\pi}>40 \mathrm{GeV}$, 
to further suppress the background rates and increase the signal-to-background ratio. This result is shown in the fourth column of Table III. To detect the $A H^{+}$signal event, we select the invariant mass of the $b \bar{b}$ pair so that it is within $10 \mathrm{GeV}$ around $M_{A}$. The result is shown in the fifth column of Table III. As shown, after these cuts, the significance of the signal event increases by a factor of 5 at the cost of about half of the number of signal events. A similar result for detecting the $\mathrm{HH}^{+}$signal event is given in Table IV] Up to now, the analysis was carried out at the parton level with a perfect measurement of the observable parton momenta. In reality, the detection efficiency of the detector cannot be one hundred percent. To estimate the effect due to the finite detection efficiency of the detector, we smear the four-momenta of the final state observable partons (excluding neutrino) by assuming a Gaussian error of $50 \% \sqrt{E}$, where $E$ is the energy of the parton. After repeating the above analysis with the basic cuts, the $E_{T}, p_{T}^{\pi}$, and $m_{b \bar{b}}$ cuts, we obtain the signal event rates as shown in the last column of Table III and Table IV for the $A H^{+}$and $H H^{+}$ channels, respectively. It is clear that the $A H^{+}$signal is much easier than the $\mathrm{HH}^{+}$signal to be identified for Case A. Furthermore, from the distribution of the $b \bar{b}$ invariant mass $m_{b \bar{b}}$, one can extract out the $A$ boson mass $M_{A}$ from its peak position, cf. Fig. 5. From the distribution of the transverse mass of $H^{+}$defined as Eq. (18), one can extract out the $H^{+}$boson mass $M_{H^{+}}$from its jacobian peak position, cf. Fig. 6. Given the measured $M_{A}$ and $M_{H^{+}}$values, one can then test the mass relation, Eq. (11), to confirm the MSSM Higgs sector. This is the conclusion for the case that a $\mathrm{AH}^{+}$signal, as predicted by Case $\mathrm{A}$, is found. If it is not found, then one can readily constrain the MSSM. For example, according to the last column in Table III, if a $A H^{+}$signal is not found, then at the two standard deviation level, the product of $B(A \rightarrow b \bar{b})$ and $B\left(H^{+} \rightarrow \nu_{\tau} \tau^{+}\right)$in the MSSM has to be less than $\frac{2 \sqrt{87}}{202} \times(0.91)(0.98)=0.082$ for $M_{A}=101 \mathrm{GeV} .{ }^{4}$ We note that, as discussed in the previous sections, this upper bound on $B(A \rightarrow b \bar{b}) \times B\left(H^{+} \rightarrow \nu_{\tau} \tau^{+}\right)$as a function of $M_{A}$ (i.e., $m_{b \bar{b}}$ ) is not sensitive to the other SUSY parameters in the MSSM.

Surely, the above bound can be improved by including the negatively charged mode $A H^{-}$of the signal event (whose rate is about half of the $A H^{+}$rate, cf. Fig. 1) and the other

\footnotetext{
${ }^{4}$ Here, we require the number of signal events to be less than two times the statistical fluctuation $(\sqrt{B})$ of the SM background events.
} 
decay mode of $\tau^{ \pm}$(such as $\tau^{+} \rightarrow \rho^{+} \bar{\nu}_{\tau}$ and $\tau^{-} \rightarrow \rho^{-} \nu_{\tau}$ ). Since the decay branching ratio $\mathrm{B}\left(\tau^{+} \rightarrow \rho^{+} \bar{\nu}_{\tau}\right)$ is a factor of 2 larger than $\mathrm{B}\left(\tau^{+} \rightarrow \pi^{+} \bar{\nu}_{\tau}\right)$, and the dominant decay mode of $\rho^{+} \rightarrow \pi^{+} \pi^{0}$ produces a $\pi^{+}$whose typical momentum is smaller than that produced in $\tau^{+} \rightarrow \pi^{+} \bar{\nu}_{\tau}$, we expect that including the $\tau^{+} \rightarrow \rho^{+} \bar{\nu}_{\tau}$ channel would increase the event rate by about a factor of 2 . Therefore, after including both the $A H^{+}$and $A H^{-}$channels with $\tau$ decays into the $\pi \nu$ or $\rho \nu$ modes, the signal rate will increase by about a factor of 3 . Assuming the background rates increase by the same factor, the resolution power will increase by a factor about $\sqrt{3}$. For example, the above constraint will read as $B(A \rightarrow b \bar{b}) \times B\left(H^{ \pm} \rightarrow\right.$ $\left.\nu_{\tau} \tau^{ \pm}\right)<0.08 / \sqrt{3}=0.046$ for $M_{A}=101 \mathrm{GeV}$ in the MSSM. However, a reliable conclusion can only be drawn from the study using a full event generator (that predicts the distributions of final state hadrons) folded with realistic detector simulation.

Secondly, we consider Case B, in which the production rates of $\mathrm{HH}^{+}$and $\mathrm{AH}^{+}$are about the same. Following the same analysis procedures as in Case A, we obtain the results shown in Table $\nabla$ and Fig. 9] According to the last column in Table $\nabla$ if a $A H^{+}$signal is not found, then at the two standard deviation level, $B(A \rightarrow b \bar{b}) \times B\left(H^{+} \rightarrow \nu_{\tau} \tau^{+}\right)$in the MSSM has to be less than $\frac{2 \sqrt{50}}{65} \times(0.90)(0.90)=0.18$ for $M_{A}=166 \mathrm{GeV}$. After including the $A H^{-}$ production channel and $\tau \rightarrow \rho \nu$ decay modes, we expect the above bound to improve by about a factor of $\sqrt{3}$, i.e., $B(A \rightarrow b \bar{b}) \times B\left(H^{ \pm} \rightarrow \nu_{\tau} \tau^{ \pm}\right)<0.18 / \sqrt{3}=0.1$ for $M_{A}=166 \mathrm{GeV}$ in the MSSM.

Thirdly, we consider Case $\mathrm{C}$, in which $H^{+}$predominantly decays into the $t \bar{b}$ mode. For the CP-odd Higgs boson decays into a $b \bar{b}$ pair, the dominant SM background comes from the $b \bar{b} t \bar{t}$ production when the decay products of $W^{-}$, produced from $\bar{t}$ decay, escape detection. The sensitivity of the LHC to this case is presented in Table VI and Figs. 10] and 11] Due to the complexity of the background simulation, the result of our parton level Monte Carlo analysis for the background event can only be viewed as an estimate. A more reliable calculation using the full event generator with detector simulation is needed to draw a definite conclusion. Nevertheless, we illustrated that the $t \bar{b}$ decay mode of the signal event can be detected at the LHC with a reasonable resolution on the determination of $M_{A}$ and $M_{H^{+}}$, cf. Figs. [10 and 11. A crude estimate from Table VI reveals that if a $A H^{+}$signal is not found, then at the two standard deviation level, $B(A \rightarrow b \bar{b}) \times B\left(H^{+} \rightarrow t \bar{b}\right)$ has to be 
TABLE VII: Numbers of $A H^{+}$signal and background events for Case A, with $M_{A}=101 \mathrm{GeV}$ in the $b \bar{b} \pi^{+} E_{T}$ channel, at the Tevatron with an integrated luminosity of $30 \mathrm{fb}^{-1}$. The $b$-tagging efficiency $(50 \%$, for tagging both $b$ and $\bar{b}$ jets) is included, and the kinematic cuts listed in each column are applied sequentially.

\begin{tabular}{c|c|c|c|c}
\hline \hline & Basic Cuts & $E_{T}>50 \mathrm{GeV}$ & $p_{T}^{\pi}>40 \mathrm{GeV}$ & $\begin{array}{c}90<M_{A}<110 \mathrm{GeV} \\
\text { or }\end{array}$ \\
\hline$A H^{+}$ & 11 & 8 & 3 & $105<M_{H}<125 \mathrm{GeV}$ \\
$H H^{+}$ & 1 & 1 & 0 & 3 \\
\hline$W b \bar{b}$ & 383 & 27 & 3 & 0 \\
$t \bar{b}$ & 6 & 3 & 0 & 0 \\
$t \bar{t}$ & 0 & 0 & 0 & 0 \\
$W g$ & 0 & 0 & 0 & 0 \\
\hline \hline
\end{tabular}

less than $\frac{2 \sqrt{3}}{18} \times(0.89)(0.79)=0.135$ for $M_{A}=250 \mathrm{GeV}$ in the MSSM.

Finally, we comment on the potential of the Fermilab Tevatron. As shown in Fig. 1, the production rate of the $A H^{+}$event at the Tevatron is only sizable for small $M_{A}$. Assuming an integrated luminosity of $30 \mathrm{fb}^{-1}$ at the Tevatron, the event yield for Case $\mathrm{A}$ is shown in Table VII. It is evident that it is challenging to detect such a signal at the Tevatron.

\section{CONCLUSIONS}

Most production processes studied in the literature for testing the MSSM contain at least two SUSY parameters ( such as $\tan \beta$ and $M_{A}$ ) in the search for supersymmetric Higgs bosons. Furthermore, the detection efficiency of the signal event depends on the assumed decay channels of the SUSY particles, hence, on the detailed choice of SUSY parameters. If the signal is not found after comparing experimental data with theory prediction, it is a common practice to constrain the product of the production cross section and the decay branching ratios of final state SUSY particles as a function of the multiple-dimension SUSY 


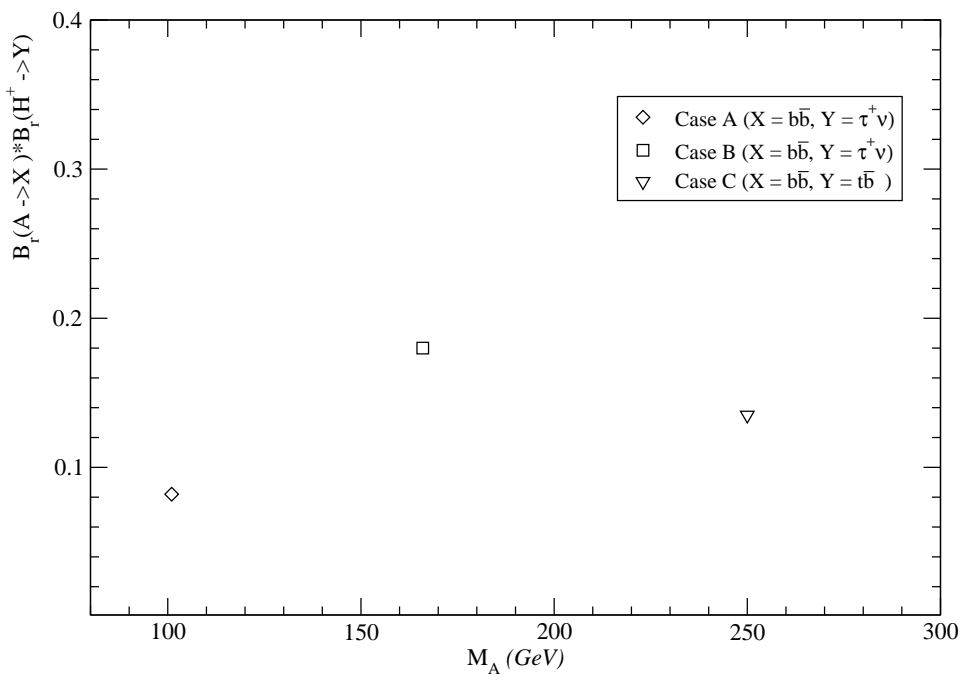

FIG. 12: Constraints on the product of branching ratios $B(A \rightarrow b \bar{b}) \times B\left(H^{+} \rightarrow \tau^{+} \nu_{\tau}\right)$ as a function of $M_{A}$ for Case A and Case B, and $B(A \rightarrow b \bar{b}) \times B\left(H^{+} \rightarrow t \bar{b}\right)$ for Case C, at the LHC, where $\tau^{+}$ decays into $\pi^{+} \bar{\nu}_{\tau}$ channel.

parameter space of the MSSM.

In Ref. 23] , a novel proposal was made to study the $A H^{ \pm}$production process at hadron colliders. It was pointed out that this process possesses the following interesting properties:

- Its Born level production rate depends only on one SUSY parameter that can be determined by kinematic variables (e.g., the invariant mass of the $b \bar{b}$ pair).

- Its higher order production rate is not sensitive to detailed SUSY parameters through electroweak radiative corrections.

- Its final state particle kinematics can be properly modelled without specifying any SUSY parameters. Hence, the detection efficiency of the signal event can be accurately determined.

- If the signal is found, it can be used to distinguish the MSSM Higgs sector from its alternatives, e.g., THDM.

- If the signal is not found, one can constrain the MSSM by limiting the product of decay branching ratios alone, without convoluting with the production cross section. 
At the LHC, the $A H^{+}$signal event can be produced from the tree level process $q \bar{q}^{\prime} \rightarrow$ $W^{+*} \rightarrow A H^{+}$, whose rate depends only on $M_{A}$ and $M_{H^{+}}$, for the coupling of $W-A-H^{+}$ is fixed by the weak gauge coupling $g$, required by $S U(2)$ gauge invariance. Furthermore, in the MSSM, $M_{A}$ and $M_{H^{+}}$are related as Eq. (11), therefore the $A H^{+}$production rate depends only on one SUSY parameter, i.e., $M_{A}$, which can be kinematically determined from experimental data by reconstructing the invariant mass of the decay particles of $A$. For example, when $A$ decays into the $b \bar{b}$ channel, the invariant mass of $b \bar{b}$ pair reveals the mass of $A$. Since both $A$ and $H^{+}$are scalar particles, the angular distributions of their decay particles can be accurately modelled, i.e., an isotropic distribution in its rest frame. If the signal is found, the mass relation, Eq. (1), can be tested to distinguish the MSSM from the THDM in which this mass relation does not generally hold. If the signal event is not found, then one can constrain the MSSM parameters by limiting the product of the decay branching ratios $B(A \rightarrow X) \times B\left(H^{+} \rightarrow Y\right)$ as a function of $M_{A}$, where $X$ and $Y$ are the decay channels of $A$ and $H^{+}$, respectively, studied by experimentalists.

In Sec. II, we show that the electroweak radiative corrections that depend on detailed SUSY parameters are usually small in comparison to the uncertainty in higher order (beyond the NLO) QCD corrections, parton distribution functions, or the accuracy of the experimental measurement.

To test whether such a signal can be detected at the LHC, we performed a Monte Carlo study at the parton level in Sec. III. We consider three cases of MSSM to cover the decay modes of $A(\rightarrow b \bar{b}) H^{+}\left(\rightarrow \tau^{+} \nu_{\tau}\right)$ and $A(\rightarrow b \bar{b}) H^{+}(\rightarrow t \bar{b})$. We concluded in the last part of Sec. III that at the LHC this signal event can indeed provide useful information about the MSSM Higgs sector. For example, if the $\mathrm{AH}^{+}$signal is not found in the decay mode of $\tau^{+} \rightarrow$ $\pi^{+} \bar{\nu}_{\tau}$, then we can constrain the product of branching ratios $B(A \rightarrow b \bar{b}) \times B\left(H^{+} \rightarrow \tau^{+} \nu_{\tau}\right)$ as a function of $M_{A}$, as shown in Fig. 12. This corresponds to Case A or Case B defined in Table II. In case $\mathrm{C}$, for $M_{H^{+}}>m_{t}+m_{b}$, not finding the signal event implies an upper bound on $B(A \rightarrow b \bar{b}) \times B\left(H^{+} \rightarrow t \bar{b}\right)$ for a given $M_{A}$, and therefore constrains the MSSM as a function of one SUSY parameter. Including the negatively charged channel $A H^{-}$and the $\rho \nu$ decay mode of $\tau$ can tighten the above bounds roughly by a factor of $\sqrt{3}$. However, to have a more accurate conclusion, a full event generator with detector simulation should be 


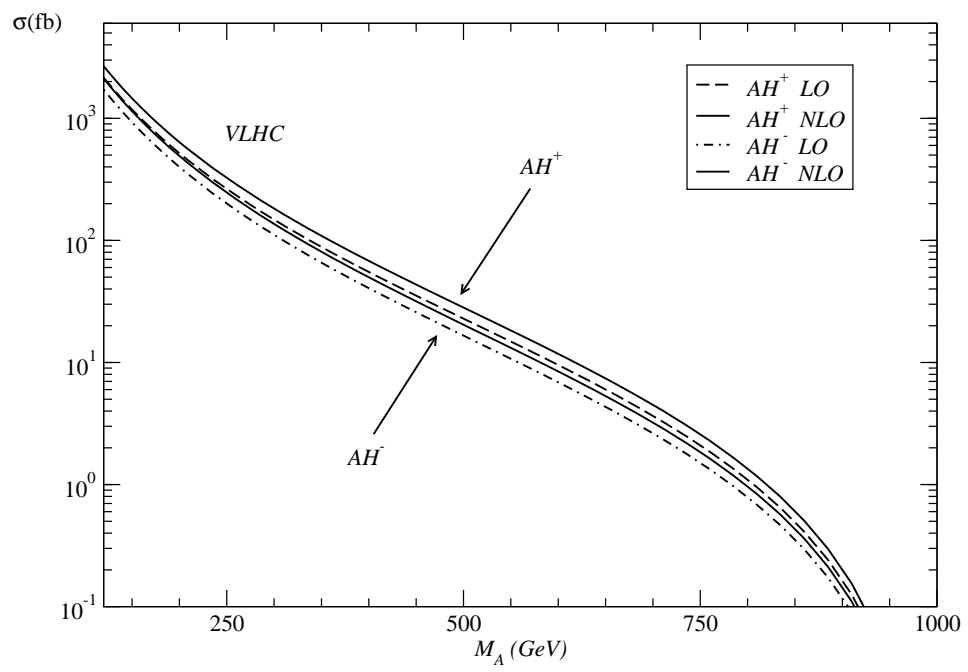

FIG. 13: The LO (dotted lines) and NLO QCD (solid lines) cross sections of the $A H^{+}$and $A H^{-}$ pairs as a function of $M_{A}$ at the VLHC (a $200 \mathrm{TeV} p p$ collider).

used to repeat the analysis outlined in this paper.

From Fig. 1, we see that the $A H^{+}$rate becomes very small (less than about $0.1 \mathrm{fb}$ ) at the LHC once $M_{A}$ is larger than $400 \mathrm{GeV}$. Hence, to cover the whole mass spectrum of the TeV scale MSSM, we should find a high energy collider that can be sensitive to this process for $M_{A}$ approaching the $\mathrm{TeV}$ region. This could be one of the motivations for proposing a future Very Large Hadron Collider (VLHC), a $200 \mathrm{TeV}$ proton-proton collider. The signal rates at the VLHC are shown in Fig. 13.

\section{Acknowledgments}

We thank K. Hagiwara, G.L. Kane, H.K. Kim, P. Nadolsky, Y. Okada, T. Tait and G. Tavares-Velasco for useful discussions. This work was supported in part by the NSF grants PHY-0244919 and PHY-0100677. 


\section{APPENDICES}

\section{A. Renormalization}

In this paper, we adopt the on-shell renormalization scheme developed by Dabelstein[26] to calculate the one-loop electroweak corrections. The standard model parameters are fixed by defining $\alpha_{e m}, m_{W}$ and $m_{Z}$, and the additional SUSY parameters in the Higgs sector ${ }^{5}$ are fixed by the following renormalization conditions: (1) the tadpole contributions $\left(T_{H_{1}}=0\right.$, $\left.T_{H_{2}}=0\right)$, (2) the on-shell condition for the mass of $A,(3)$ the on-shell condition for the wavefunction of $A$, (4) a renormalization condition on $\tan \beta$ (which requires $\delta v_{1} / v_{1}=\delta v_{2} / v_{2}$ ), and (5) a vanishing $A-Z$ mixing for an on-shell $A$.

\section{B. Calculation of $F^{(1)}\left(q^{2}\right)$}

The one-loop correction to the renormalized form factor of the $W^{ \pm} H^{\mp} A$ vertex, apart from the effective weak gauge coupling $\bar{g}$, can be written as

$$
\begin{aligned}
F^{(1)}\left(q^{2}\right)= & \tilde{Z}_{A A}^{1 / 2} \tilde{Z}_{H^{+} H^{-}}^{1 / 2}\left\{1+\delta F_{W H A}\right. \\
& \left.+F_{W H A}^{1 \mathrm{PI}}\left(M_{A}^{2}, M_{H}^{2}, q^{2}\right)\right\}-1
\end{aligned}
$$

where $\tilde{Z}_{A A}$ and $\tilde{Z}_{H^{+} H^{-}}$are the finite wavefunction factors for the renormalization of the external Higgs bosons $A$ and $H^{ \pm}$. In our scheme,

$$
\begin{aligned}
\tilde{Z}_{A A} & =1 \\
\tilde{Z}_{H^{+} H^{-}} & =1-\Pi_{H^{+} H^{-}}^{\prime}\left(M_{A}^{2}+m_{W}^{2}\right)+\Pi_{A A}^{\prime}\left(M_{A}^{2}\right),
\end{aligned}
$$

where $\Pi_{A A}^{\prime}\left(M_{A}^{2}\right)$ denotes taking the derivative of the two point function $\Pi_{A A}\left(k^{2}\right)$ of the CP-odd scalar $A$ with respect to $k^{2}$ at $k^{2}=M_{A}^{2}$, etc. The terms inside the curly bracket of Eq. (22) arise from the renormalized vertex function of $W H A . F_{W H A}^{1 \mathrm{PI}}\left(p_{A}^{2}, p_{H}^{2}, q^{2}\right)$ represents

\footnotetext{
5 There are 7 parameters in the Higgs sector of the MSSM. They are $g^{\prime}, g, v_{1}, v_{2}, m_{1}, m_{2}$, and $m_{3}$. Beyond the Born level, the wavefunction renormalization factors $Z_{H_{1}}$ and $Z_{H_{2}}$ also need to be introduced to renormalize the theory, where $H_{1}$ and $H_{2}$ denote the two Higgs doublets in the model.
} 
the one-loop contribution of the one-particle-irreducible (1PI) diagrams with $p_{A}^{2}, p_{H}^{2}, q^{2}$ as the four-momentum square of the incoming $A, H^{\mp}$ and $W^{ \pm}$particles, respectively. $\delta F_{W H A}$ is the counterterm contribution resulting from the field renormalization of $H^{+}$and $A$ :

$$
H^{+} A \rightarrow H^{+} A\left(1+\frac{1}{2} \delta Z_{H^{+}}+\frac{1}{2} \delta Z_{A}\right)
$$

In terms of the independent counterterms fixed by the renormalization scheme, the wavefunction counterterms $\delta Z_{H^{+}}$and $\delta Z_{A}$ can be written as $\left(\sin ^{2} \beta\right) \delta Z_{H_{1}}+\left(\cos ^{2} \beta\right) \delta Z_{H_{2}}$ which is found to be equal to $-\frac{1}{2} \Pi_{A A}^{\prime}\left(M_{A}^{2}\right)$. We note that in $\delta F_{W H A}$ the contributions from the counterterms of the weak gauge coupling and the wavefunction renormalization of the $W$ boson are not included, because they should be combined with the $W$-boson self energy contribution to derive the running weak gauge coupling $\bar{g}\left(q^{2}\right)$. In our numerical calculation, we use

$$
\bar{g}^{2}=4 \sqrt{2} m_{W}^{2} G_{F}
$$

In summary, the one-loop electroweak correction to $F^{(1)}\left(q^{2}\right)$ is found to be

$$
\begin{aligned}
& F^{(1)}\left(q^{2}\right) \equiv F_{W H A}^{1 \mathrm{PI}}\left(M_{A}^{2}, M_{H^{ \pm}}^{2}, q^{2}\right) \\
& -\frac{1}{2} \Pi_{H^{+} H^{-}}^{\prime}\left(M_{A}^{2}+m_{W}^{2}\right)-\frac{1}{2} \Pi_{A A}^{\prime}\left(M_{A}^{2}\right) .
\end{aligned}
$$

In the above equation, the top- and bottom-loop contribution to $F_{W H A}^{1 \mathrm{PI}}$ is given by

$$
\begin{aligned}
& F_{W H A}^{1 \mathrm{PI}(\text { quark })}\left(q^{2}, p_{A}^{2}, p_{H}^{2}\right) \\
& =\sum_{f f f^{\prime}=t t b, b b t} F_{W H A}^{f f f^{\prime}}\left(q^{2}, p_{A}^{2}, p_{H}^{2}\right),
\end{aligned}
$$

with

$$
\begin{aligned}
& F_{W H A}^{f f f^{\prime}}\left(p_{A}^{2}, p_{H}^{2}, q^{2}\right)=+\frac{N_{c}}{16 \pi^{2}} y_{f}^{2}\left\{p_{A}^{2} C_{31}^{f f f^{\prime}}-p_{H}^{2} C_{32}^{f f f^{\prime}}\right. \\
& +\left(2 p_{A} \cdot p_{H}-p_{A}^{2}\right) C_{33}^{f f f^{\prime}}-\left(2 p_{A} \cdot p_{H}-p_{H}^{2}\right) C_{34}^{f f f^{\prime}} \\
& +(D+2)\left(C_{35}^{f f f^{\prime}}-C_{36}^{f f f^{\prime}}\right)+p_{A}^{2} C_{21}^{f f f^{\prime}}-\left(2 p_{A} \cdot p_{H}\right. \\
& \left.+p_{H}^{2}\right) C_{22}^{f f f^{\prime}}-2 p_{A}^{2} C_{23}^{f f f^{\prime}}-(D-2) C_{24}^{f f f^{\prime}}-m_{f}^{2} C_{11}^{f f f^{\prime}} \\
& \left.-\left(q^{2}+m_{f}^{2}\right) C_{12}^{f f f^{\prime}}\right\}-c_{f} \frac{1}{16 \pi^{2}} y_{f} y_{f^{\prime}} m_{f} m_{f^{\prime}} C_{0}^{f f f^{\prime}} .
\end{aligned}
$$

where $c_{f}=+1$ and -1 for $f f f^{\prime}=t t b$ and $b b t$, respectively, and $C_{i j}^{f f f^{\prime}}$ are defined in terms of the Passarino-Veltman functions 37] with

$$
C_{i j}^{f f f^{\prime}}=C_{i j}\left(p_{A}^{2}, p_{H}^{2},\left(p_{A}+p_{H}\right)^{2} ; m_{f}, m_{f}, m_{f^{\prime}}\right) .
$$


The stop- and sbottom-loop contribution is given by

$$
\begin{aligned}
& F_{W H A}^{1 \mathrm{PI}(\mathrm{squark})}\left(p_{A}^{2}, p_{H}^{2}, q^{2}\right)=\frac{N_{c}}{16 \pi^{2}} \sqrt{2} \sum_{i, j, k=1}^{2} \\
& \times\left\{U_{i L}^{*} D_{L k}\left(i \lambda\left[\tilde{t}_{j}^{*}, \tilde{t}_{i}, A\right]\right) \lambda\left[\tilde{b}_{k}^{*}, \tilde{t}_{j}, H^{-}\right] \tilde{C}^{\tilde{t}_{i} \tilde{t}_{j} \tilde{b}_{k}}\right. \\
& \left.-U_{k L}^{*} D_{L i}\left(i \lambda\left[\tilde{b}_{i}^{*}, \tilde{b}_{j}, A\right]\right) \lambda\left[\tilde{b}_{j}^{*}, \tilde{t}_{k}, H^{-}\right] \tilde{C}^{\tilde{b}_{i} \tilde{b}_{j} \tilde{t}_{k}}\right\},
\end{aligned}
$$

where $U_{I i}, D_{I i}$ are the rotation matrices for stops and sbottoms between the weak eigenstate basis $(I=L, R)$ and the mass eigenstate basis $(i=1,2)$, respectively. $\lambda\left[\tilde{f}_{i}^{*}, \tilde{f}_{j}^{\prime}, \phi_{k}\right]$ represents the coefficient of the $\tilde{f}_{i}^{*} \tilde{f}_{j}^{\prime} \phi_{k}$ interaction in the MSSM Lagrangian, as listed in Appendix C, and

$$
\tilde{C}^{\tilde{f}_{i} \tilde{f}_{j}^{\prime} \tilde{f}_{k}^{\prime \prime}}=\left(C_{11}-C_{12}\right)\left(p_{1}^{2}, p_{2}^{2}, q^{2} ; m_{\tilde{f}_{i}}, m_{\tilde{f}_{j}^{\prime}}, m_{\tilde{f}_{k}^{\prime \prime}}\right) .
$$

The quark (top and bottom) and squark (stop and sbottom) loop contributions to the selfenergies $\Pi_{A A}\left(q^{2}\right)$ and $\Pi_{H^{+} H^{-}}\left(q^{2}\right)$ can be found in Appendix $\mathbf{E}$.

\section{Squark couplings with $H^{ \pm}$and $A$}

The mass eigenstates of the squarks are related to their weak eigenstates by the rotation matrix $O_{i I}^{f \dagger}$ with $\tilde{f}_{i}=\sum_{I} O_{i I}^{f \dagger} \tilde{f}_{I}$, where $i=1,2$ and $I=L, R ; O_{I i}^{f}=U_{I i}$ and $D_{I i}$ for $f=t$ and $b$, respectively. In terms of the mixing angles $\theta_{f}$, we have

$$
\left(\begin{array}{c}
\tilde{f}_{1} \\
\tilde{f}_{2}
\end{array}\right)=\left(\begin{array}{cc}
\cos \theta_{f} & \sin \theta_{f} \\
-\sin \theta_{f} & \cos \theta_{f}
\end{array}\right)\left(\begin{array}{c}
\tilde{f}_{L} \\
\tilde{f}_{R}
\end{array}\right) .
$$

Here, we define the mixing angle $\theta_{f}$ so that $\tilde{f}_{1}$ is lighter than $\tilde{f}_{2}$.

The coupling constants among the weak-eigenstate squarks and the Higgs bosons are defined through the Lagrangian

$$
\mathcal{L}=\cdots+\lambda\left[\tilde{f}_{I}^{*}, \tilde{f}_{J}^{\prime}, \phi, \ldots\right] \tilde{f}_{I}^{*} \tilde{f}_{J}^{\prime} \phi, \ldots+\cdots
$$

Hence, the coupling constants for the mass-eigenstate squarks are a linear combination of the couplings for the weak-eigenstate squarks, and

$$
\begin{aligned}
\lambda\left[\tilde{f}_{i}^{*}, \tilde{f}_{j}^{\prime}, \phi, \ldots\right] & =\lambda\left[\tilde{f}_{I}^{*} O_{I i}^{f}, O_{i I}^{f^{\prime} \dagger} \tilde{f}_{J}^{\prime}, \phi, \ldots\right] \\
& =O_{I i}^{f} O_{i I}^{f^{\prime} \dagger} \lambda\left[\tilde{f}_{I}^{*}, \tilde{f}_{J}^{\prime}, \phi, \ldots\right] .
\end{aligned}
$$


The relevant couplings $\lambda\left[\tilde{f}_{I}^{*}, \tilde{f}_{J}^{\prime}, \phi, \ldots\right]$, denoted as $\lambda_{\tilde{f}_{I}^{*} \tilde{f}_{J}^{\prime} \phi, . .}$, are listed below.

$$
\begin{aligned}
& \lambda_{\tilde{b}_{L}^{*} \tilde{L}_{L} H^{-}}=\frac{-\sqrt{2}}{v}\left(m_{W}^{2} \sin 2 \beta-m_{b}^{2} \tan \beta-m_{t}^{2} \cot \beta\right), \\
& \lambda_{\tilde{b}_{L}^{*} \tilde{t}_{R} H^{-}}=\frac{\sqrt{2} m_{t}}{v}\left(A_{t} \cot \beta+\mu\right), \\
& \lambda_{\tilde{b}_{R}^{*} \tilde{t}_{L} H^{-}}=\frac{\sqrt{2} m_{b}}{v}\left(A_{b} \tan \beta+\mu\right), \\
& \lambda_{\tilde{b}_{R}^{*} \tilde{t}_{R} H^{-}}=\frac{2 \sqrt{2} m_{t} m_{b}}{v \sin 2 \beta}, \\
& \lambda_{\tilde{f}_{L}^{*} \tilde{f}_{L} A}=\lambda_{\tilde{f}_{R}^{*} \tilde{f}_{R} A}=0,(\tilde{f}=\tilde{t}, \tilde{b}), \\
& \lambda_{\tilde{b}_{L}^{*} \tilde{b}_{R} A}=\frac{i m_{b}}{v}\left(A_{b} \tan \beta+\mu\right), \\
& \lambda_{\tilde{t}_{L}^{*} \tilde{t}_{R} A}=\frac{i m_{t}}{v}\left(A_{t} \cot \beta+\mu\right), \\
& \lambda_{\tilde{b}_{L}^{*} \tilde{b}_{L} A A}=\frac{-m_{b}^{2}}{v^{2}} \tan ^{2} \beta+\frac{g_{Z}^{2}}{4}\left(T_{b}-Q_{b} s_{W}^{2}\right) \cos 2 \beta, \\
& \lambda_{\tilde{b}_{R}^{*} \tilde{b}_{R} A A}=\frac{-m_{b}^{2}}{v^{2}} \tan ^{2} \beta+\frac{g_{Z}^{2}}{4} Q_{b} s_{W}^{2} \cos 2 \beta, \\
& \lambda_{\tilde{b}_{L}^{*} \tilde{b}_{L} A A}=\frac{-m_{t}^{2}}{v^{2}} \cot ^{2} \beta+\frac{g_{Z}^{2}}{4}\left(T_{t}-Q_{t} s_{W}^{2}\right) \cos 2 \beta, \\
& \lambda_{\tilde{b}_{R}^{*} \tilde{b}_{R} A A}=\frac{-m_{t}^{2}}{v^{2}} \cot ^{2} \beta+\frac{g_{Z}^{2}}{4} Q_{t} s_{W}^{2} \cos 2 \beta, \\
& \lambda_{\tilde{b}_{L}^{*} \tilde{b}_{L} H^{+} H^{-}}=\frac{-2 m_{t}^{2}}{v^{2}} \tan ^{2} \beta+\frac{g_{Z}^{2}}{2}\left(T_{t}-Q_{t} s_{W}^{2}\right) \cos 2 \beta, \\
& \lambda_{\tilde{b}_{R}^{*} \tilde{b}_{R} H^{+} H^{-}}=\frac{-2 m_{b}^{2}}{v^{2}} \tan ^{2} \beta+\frac{g_{Z}^{2}}{2} Q_{b} s_{W}^{2} \cos 2 \beta, \\
& \lambda_{\tilde{t}_{L}^{*} \tilde{t}_{L} H^{+} H^{-}}=\frac{-2 m_{b}^{2}}{v^{2}} \tan ^{2} \beta+\frac{g_{Z}^{2}}{2}\left(T_{b}-Q_{t} s_{W}^{2}\right) \cos 2 \beta, \\
& \lambda_{\tilde{t}_{R}^{*} \tilde{t}_{R} H^{+} H^{-}}=\frac{-2 m_{t}^{2}}{v^{2}} \cot ^{2} \beta+\frac{g_{Z}^{2}}{2} Q_{t} s_{W}^{2} \cos 2 \beta,
\end{aligned}
$$

where $T_{t}, T_{b}, Q_{t}$ and $Q_{b}$ are $\frac{1}{2}, \frac{-1}{2}, \frac{2}{3}$ and $\frac{-1}{3}$, respectively, and

$$
\begin{gathered}
\lambda_{\tilde{t}_{I}^{*} \tilde{b}_{J} H^{+}}=\lambda_{\tilde{b}_{I}^{*} \tilde{t}_{J} H^{-}}, \\
\lambda_{\tilde{f}_{R}^{*} \tilde{f}_{L} A}=-\lambda_{\tilde{f}_{L}^{*} \tilde{f}_{R} A},
\end{gathered}
$$

for $I, J=L, R$ and $\tilde{f}=\tilde{t}, \tilde{b}$. 


\section{Squark contributions to the $\rho$ parameter}

The squark one-loop contribution to the $\rho$ parameter is given by

$$
\Delta \rho=\rho-1=-4 \sqrt{2} G_{F} \operatorname{Re}\left[\Delta \Pi_{T}^{11}(0)-\Delta \Pi_{T}^{33}(0)\right]
$$

with [39]

$$
\begin{gathered}
\Delta \Pi_{T}^{11}\left(q^{2}\right)=\frac{N_{c}}{16 \pi^{2}} \sum_{f=t, b} \sum_{i, j=1}^{2} T_{f_{L}}{ }^{2}\left|O_{L i}^{f}\right|^{2}\left|O_{L j}^{f}\right|^{2} \\
\times B\left(q^{2} ; m_{\tilde{f}_{i}}^{2}, m_{\tilde{f}_{j}}^{2}\right), \\
\Delta \Pi_{T}^{33}\left(q^{2}\right)=\frac{N_{c}}{32 \pi^{2}} \sum_{i, j=1}^{2}\left|U_{L i}\right|^{2}\left|D_{L j}^{f}\right|^{2} B\left(q^{2} ; m_{\tilde{u}_{i}}^{2}, m_{\tilde{d}_{j}}^{2}\right),
\end{gathered}
$$

where $O_{I i}^{t}=U_{I i}$ and $O_{I i}^{b}=D_{I i} ; B\left(q^{2} ; m_{1}^{2}, m_{2}^{2}\right) \equiv A\left(m_{1}^{2}\right)+A\left(m_{2}^{2}\right)-4 B_{22}\left(q^{2} ; m_{1}^{2}, m_{2}^{2}\right)$. By using the expression

$$
B\left(0 ; m_{1}^{2}, m_{2}^{2}\right)=-\frac{1}{2}\left(m_{1}^{2}+m_{2}^{2}\right)+\frac{m_{1}^{2} m_{2}^{2}}{m_{1}^{2}-m_{2}^{2}} \ln \frac{m_{2}^{2}}{m_{1}^{2}} .
$$

Eq. (9) is deduced under the assumption that $M^{2}=M_{Q}^{2} \simeq M_{U}^{2} \simeq M_{D}^{2} \gg m_{t}^{2}$ and the stop mixing is large $\left(m_{t}\left|X_{t}\right| \simeq M^{2}\right.$ and $\left.m_{b}\left|X_{b}\right| \simeq 0\right)$, so that $m_{\tilde{t}_{1}} \sim \mathcal{O}\left(m_{Z}\right)$, which yields $m_{\tilde{t}_{2}} \sim \sqrt{2} M$, and $m_{\tilde{b}_{1}} \sim m_{\tilde{b}_{2}} \sim M$.

\section{E. Self energies}

The (top and bottom) quark-loop contributions to the self-energies $\Pi_{A A}\left(q^{2}\right)$ and $\Pi_{H^{+} H^{-}}\left(q^{2}\right)$ in $D$-dimensions are expressed in terms of the Passarino-Veltman functions [37] by

$$
\begin{aligned}
& \Pi_{A A}^{\text {quark }}\left(q^{2}\right)=-\frac{N_{c}}{16 \pi^{2}} \sum_{f=t, b} 2 y_{f}^{2}\left\{q ^ { 2 } \left(B_{1}\left(q^{2}, m_{f}, m_{f}\right)\right.\right. \\
& \left.+B_{21}\left(q^{2}, m_{f}, m_{f}\right)\right)+D B_{22}\left(q^{2}, m_{f}, m_{f}\right) \\
& \left.+m_{f}^{2} B_{0}\left(q^{2}, m_{f}, m_{f}\right)\right\} \\
& \Pi_{H^{+} H^{-}}^{\text {quark }}\left(q^{2}\right)=-\frac{N_{c}}{16 \pi^{2}} 2\left(y_{b}^{2}+y_{t}^{2}\right)\left\{q ^ { 2 } \left(B_{1}\left(q^{2}, m_{b}, m_{t}\right)\right.\right. \\
& \left.\left.+B_{21}\left(q^{2}, m_{b}, m_{t}\right)\right)+D B_{22}\left(q^{2}, m_{b}, m_{t}\right)\right\}, \\
& -\frac{N_{c}}{16 \pi^{2}} 4 y_{b} y_{t} m_{b} m_{t} B_{0}\left(q^{2}, m_{b}, m_{t}\right) .
\end{aligned}
$$


The stop- and sbottom-loop contributions are given by

$$
\begin{aligned}
& \Pi_{A A}^{\text {squark }}\left(q^{2}\right)=-\frac{N_{c}}{16 \pi^{2}} \sum_{\tilde{f}=\tilde{t}, \tilde{b}} \sum_{i, j=1}^{2} \\
& \times \lambda\left[\tilde{f}_{i}^{*}, \tilde{f}_{j}, A\right] \lambda\left[\tilde{f}_{j}^{*}, \tilde{f}_{i}, A\right] B_{0}\left(q^{2}, m_{\tilde{f}_{i}}, m_{\tilde{f}_{j}}\right) \\
& -\frac{N_{c}}{16 \pi^{2}} 2 \sum_{\tilde{f}=\tilde{t}, \tilde{b}} \sum_{i=1}^{2} \lambda\left[\tilde{f}_{i}^{*}, \tilde{f}_{i}, A, A\right] A\left(m_{\tilde{f}_{i}}\right), \\
& \Pi_{H^{+} H^{-}}^{\text {squark }}\left(q^{2}\right)=-\frac{N_{c}}{16 \pi^{2}} \sum_{i, j=1}^{2} \\
& \times \lambda\left[\tilde{t}_{i}^{*}, \tilde{b}_{j}, H^{+}\right] \lambda\left[\tilde{b}_{j}^{*}, \tilde{t}_{i}, H^{-}\right] B_{0}\left(q^{2}, m_{\tilde{t}_{i}}, m_{\tilde{b}_{j}}\right) \\
& -\frac{N_{c}}{16 \pi^{2}} \sum_{\tilde{f}=\tilde{t}, \tilde{b}} \sum_{i=1}^{2} \lambda\left[\tilde{f}_{i}^{*}, \tilde{f}_{i}, H^{+}, H^{-}\right] A\left(m_{\tilde{f}_{i}}\right) .
\end{aligned}
$$

The self-energy $\Pi_{W W}\left(q^{2}\right)$ of the $W$ boson was already presented in the literature. For example, the quark-loop contribution can be found in Ref. [38], and the squark-loop contribution in Ref. [39].

[1] J.F. Gunion, H.E. Haber, G.L. Kane and S. Dawson, The Higgs Hunters Guide (AddisonWesley).

[2] Y. Okada, M. Yamaguchi and T. Yanagita, Prog. Theor. Phys.85 (1991) 1, J. Ellis, G. Ridolfi and F. Zwirner, Phys. Lett. B 257 (1991) 83, H.E. Haber and R. Hempfling, Phys. Rev. Lett. 66 (1991) 1815; M. Carena, M. Quiros and C. E. M. Wagner, Nucl. Phys. B 461 (1996) 407; H. E. Haber, R. Hempfling and A. H. Hoang, Z. Phys. C 75 (1997) 539; S. Heinemeyer, W. Hollik and G. Weiglein, Euro. Phys. J. C 9 (1999) 343; M. Carena, H. E. Haber, S. Heinemeyer, W. Hollik, C. E. M. Wagner and G. Weiglein, Nucl. Phys. B 580 (2000) 29; J. R. Espinosa and R. J. Zhang, Nucl. Phys. B 586 (2000) 3; G. Degrassi, P. Slavich and F. Zwirner, Nucl. Phys. B 611 (2001) 403.

[3] N. Cabbibo et al., Nucl. Phys. B 158 (1976) 295, M. Lindner, Phys. Lett. B 31 (1986) 295.

[4] M. Quiros, hep-ph/9609392. 
[5] S. Kanemura, T. Kasai and Y. Okada, Phys. Lett. B 471 (1999) 182, S. Kanemura, T. Kasai, G.-L. Lin, Y. Okada, J.-J., Tseng, and C.-P. Yuan, Phys. Rev. D 64 (2001) 053007.

[6] ATLAS Collaboration, Technical Design Report, No. CERN /LHCC/99-14/15; CMS collaboration, CERN/LHCC 97-31, CMS TDR 2, 20 June 1997.

[7] A. Djouadi et al., Higgs Working Group Summary Report of the 1st Les Houches Workshop on Physics at TeV Colliders, France (1999), hep-ph/0002258; D. Cavalli et al., Higgs Working Group Summary Report of the 2nd Les Houches Workshop on Physics at TeV Colliders, France (2001), hep-ph/0203056 E. Richter-Was, D. Froidevaux, F. Gianotti, L. Poggioli, D. Cavalli, and S. Resconi, Int. J. Mod. Phys. A 13 (1998) 1371.

[8] M. Drees, D. Roy, Phys. Lett. B 269 (1991) 155; C.-P. Yuan and T.C. Yuan Phys. Rev. D 44 (1991) 3603; J. Liu, Y.-P. Yao, Phys. Rev. D 46 (1992) 5196; A. Jimenez, J. Sola, Phys. Lett. B 389 (1996) 531.

[9] E. Eichiten, I. Hinchliffe, K. Lane, and C. Quigg, Rev. Mod. Phys. 56 (1984) 579; Addendumibid. 58 (1986) 1065.

[10] S.S.D. Willenbrock, Phys. Rev. D 35 (1987) 173; A. Krause, T. Plehn, M. Spira, and P.M. Zerwas, Nucl. Phys. B 519 (1998) 85; A. Belyaev, M. Drees, O.J.P. Eboli, J.K. Mizukoshi and S.F. Novaes, Phys. Rev. D 60 (1999) 075008; A. Belyaev, M. Drees, J.K. Mizukoshi, Euro. Phys. J. C 17 (2000) 337; A.A. Barientos Bendezu and B.A. Kniehl Nucl. Phys. B 568 (2000) 305; O. Brain and W. Hollik, Euro. Phys. J. C 13 (2000) 175; Jiang et al., Commum. Thor. Phys. 36 (2001) 61; J. Physics. G 23 (1997) 385.

[11] S. Moretti, J. Phys. G 28 (2002) 2567.

[12] J.F. Gunion, H.E. Haber, F.E. Paige, W.-K. Tung, and S.S.D. Willenbrock, Nucl. Phys. B 294 (1987) 621; Li.G. Jin, C.S. Li, R.J. Oakes, S.H. Zhu, Phys. Rev. D 62 (2000) 053008; S.H. Zhu, Phys. Rev. D 67 (2003) 075226.

[13] S. Moretti and K. Odagiri, Phys. Rev. D 55 (1997) 5627.

[14] D.A. Dicus, J.L. Hewett, C. Kao, and T.G. Rizzo, Phys. Rev. D 40 (1989) 787; A.A. Barientos Bendezu and B.A. Kniehl Phys. Rev. D 59 (1999) 015009; Phys. Rev. D 61 (2000) 097701; Phys. Rev. D 63 (2001) 015009; O. Brein, W. Hollik, and S. Kanemura, Phys. Rev. D 63 (2001) 095001. 
[15] V. Barger, R.J.N. Phillips and D.P. Roy, Phys. Lett. B 324 (1994) 236; J.F. Gunion, Phys. Lett. B 322 (1994) 125; K. Odagiri, Phys. Lett. B 452 ((1999)) 327; J.A. Coarasa, et al., Phys. Lett. B 425 (1999) 329; D.J. Miller, S. Moretti, D.P. Roy and W.J. Stirling, Phys. Rev. D 61 (2000) 055011; P. Salmi, R. Kinnunen, N. Stepanov, hep-ph/0301166.

[16] K. Odagiri, preprint RAL-TR-1999-012, February 1999, hep-ph/9901432 A. Tricomi, Talk given at 36th Rencontres de Moriond on QCD and Hadronic Interactions, Les Arcs, France, 17-24 Mar 2001, hep-ph/0105199.

[17] S. Moretti, W.J. Stirling, Phys. Lett. B 347 (1995) 291; E. Barrades, J.L. Diaz-Cruz, A. Guitierrez and A. Rosado, Phys. Rev. D 53 (1996) 1678; A. Djouadi, J. Kalinowski and P.M. Zerwas, Z. Phys. C 70 (1996) 435; E. Ma, D.P. Roy, and J. Wudka, Phys. Rev. Lett. 80 (1999) 1162; Phys. Lett. B 481 (2000) 49; S. Moretti, Phys. Lett. B 481 (2000) 49.

[18] C. Balazs, J.L. Diaz-Cruz, H.J. He, T. Tait and C.-P. Yuan, Phys. Rev. Lett. 80 (1998) 4641; Phys. Rev. D 59 (1999) 055016; M. Carena, S. Mrenna and C.E.M. Wagner, Phys. Rev. D 60 (1999) 075010.

[19] H. Bauer, M. Bisset, C. Kao, and X. Tata, Phys. Rev. D 46 (1992) 1067.

[20] H. Bauer, C. Kao, and X. Tata, Phys. Lett. B 303 (1993) 284.

[21] C. Kao and N. Stefanov, Phys. Rev. D 52 (1995) 5025.

[22] Z. Kunszt and F. Zwirner, Nucl. Phys. B 385 (1992) 3; S. Moretti, D.P. Roy, Phys. Lett. B 545 (2002) 329.

[23] S. Kanemura and C.-P. Yuan, Phys. Lett. B 530 (2002) 188.

[24] A.G. Akeroyd and S. Baek, PLB 525 (2002) 315.

[25] H.L. Lai et al., JHEP 07 (2002) 012.

[26] A. Dabelstein, Z. Phys. C 67 (1995) 495; Nucl. Phys. B 456 (1995) 25.

[27] J.F. Gunion and H.E. Haber, Nucl. Phys. B 272 (1986) 1; Erratum-ibid. Nucl. Phys. B 402 (1993) 567.

[28] Particle Data Group, Euro. Phys. J. C 15 (2000) 1.

[29] J. Erler, hep-ph/9903449.

[30] H.E. Haber, R. Hempfling and A. Hoang, Z. Phys. C 75 (1997) 539.

[31] M. Carena, J.R Espinosa, M. Quirós and C.E.M. Wagner, Phys. Lett. B 355 (1995) 209. 
[32] A.Djouadi, J.Kalinowski and M.Spira, Comput.Phys.Commun. 108 (1998) 56.

[33] The ALEPH, DELPHI, L3 and OPAL Collaborations, and the LEP Higgs Working Group, hep-ex/0107030.

[34] Higgs Working Group Collaboration (Marcela Carena et al.), hep-ph/0010338.

[35] B.P. Kersevan and E.Richter-Was, Comput. Phys. Commun. 149 (2003) 142.

[36] M. Beneke et al., hep-ph/0003033, section 9.

[37] G. Passarino and M. Veltman, Nucl. Phys. B 160 (151) 1979; For notation, we used Minkowski metric as in G.J. van Oldenborgh, Comput. Phys. Commun. 66 (1991) 1.

[38] K. Hagiwara, D. Haidt, C.S. Kim and S. Matsumoto, Euro. Phys. J. C 64 (1994) 559.

[39] For instance, the squark-loop contributions to the gauge boson two-point functions, from which $\rho$ parameter is calculated, are seen in S. Alam et al., Phys. Rev. D 62 (2000) 095011; G.C. Cho and K. Hagiwara, Nucl. Phys. B 574 (623) 623.

[40] G. L. Kane and C. P. Yuan, Phys. Rev. D 40, 2231 (1989). 